\title{
Ausgewählte Anwendungen von Stoßproblemen
}

Nach der Darstellung der dynamischen und kontaktmechanischen Grundlagen und der ausführlichen Untersuchung des Stoßproblems unter verschiedenen Bedingungen stehen im folgenden Kapitel Anwendungsbereiche aus Physik, Technik und Medizin im Mittelpunkt, für die die in den früheren Kapiteln gezeigten Ergebnisse von Bedeutung sind.

Die Gebiete, in denen die gezeigten kontaktmechanischen Grundlagen und Lösungen von Stoßproblemen Relevanz haben, sind teilweise selbst riesige Forschungszweige, die in jeweils kurzen Unterkapiteln natürlich nicht annähernd erschöpfend dargestellt werden können. Der Stil des folgenden Kapitels unterscheidet sich daher von dem der früheren Teile dieses Buches: Die behandelten Themen werden nicht mehr umfassend und mathematisch detailliert entwickelt; stattdessen wird ,nur“ beschrieben, welche Fragestellungen in welchen Anwendungsgebieten auftreten und wie man eventuell die in den früheren Kapiteln hergeleiteten Ergebnisse zur Behandlung dieser Fragen verwenden kann.

Die ersten beiden Teile des Kapitels sind der Schädigung von Systemen durch stoßartige Belastungen und stoßbasierten Testverfahren, z. B. zur Bestimmung von Materialeigenschaften, gewidmet. Der anschließende Block der Abschn. 8.3 und 8.4 beschäftigt sich mit granularen Medien - deren Dynamik durch eine Vielzahl einzelner Kollisionen bestimmt wird - und ihren astrophysikalischen Anwendungen. Zwei Unterkapitel zu den Themen Sport und Medizin beschließen den Hauptteil dieses Buches.

Diese Klassifikation möglicher Anwendungen der Mechanik von Kollisionen ist nicht immer eindeutig; Überschneidungen existieren unter anderem zwischen den Bereichen Sport und Medizin oder zwischen der theoretischen Beschreibung und den Anwendungen granularer Medien. Auch Testverfahren haben in der Regel einen bestimmten praktischen Hintergrund; so könnten Prüfverfahren zur Bestimmung der viskoelastischen Eigenschaften von Gelenkknorpel ebenso gut in dem Unterkapitel beschrieben werden, das sich medizinischen Anwendungen widmet.

Wer an mögliche Anwendungen von Stoßproblemen denkt, wird früher oder später bei der Auslegung von Schutzsystemen landen. Öffentliche, frei verfügbare Forschungsergebnisse 
zur Verbesserung von Schutzsystemen führen allerdings sehr wahrscheinlich in erheblichem Maße auch zur Verbesserung von Mitteln, diese Schutzsysteme zu durchbrechen. Forschung auf diesem Gebiet widerspricht daher der Zivilklausel der Hochschule, an der dieses Buch entstanden ist, der Technischen Universität Berlin, und wird entsprechend nicht in dem folgenden Kapitel behandelt.

\subsection{Schlagverschleiß}

Schlagverschlei $\aleph^{1}$ durch die fortgesetzte stoßartige Einwirkung mit Festkörper-Teilchen ist eine wesentliche Quelle der Schädigung von festen Oberflächen, beispielsweise im Bergbau [1]. Häufig sind dabei die erodierenden Partikel aus einem härteren Material als die Oberfläche und daher abrasive Verschleißmechanismen, wie Mikroschneiden und -pflügen, dominant. Es kommt aber auch zu plastischer Deformation, Ermüdung [2] und bei spröden Oberflächen zu verschiedenen Formen des Bruches. Bei sehr großen Stoßgeschwindigkeiten treten darüber hinaus hohe Blitztemperaturen im Kontakt auf [3], die die Festigkeit herabsetzen und chemo-mechanische Verschleißformen initiieren können.

Neben der umfangreichen Literatur zum Schlagverschleiß von Metallen und Keramiken gibt es mehrere Arbeiten zur entsprechenden Schädigung von Polymeren [4], Elastomeren [5], Faserverbundwerkstoffen [6] oder Thermoplasten [7]. Diese Materialklassen haben teilweise eigene Schadensmechanismen, die die Abhängigkeiten der Verschleißintensität von den Stoßparametern beeinflussen; in Faserverbundwerkstoffen spielt beispielsweise der Stoßwinkel relativ zur Orientierung der Fasern eine wesentliche Rolle [6].

Wegen der vergleichsweise einfachen Mechanismen des abrasiven Verschleißes gibt es mehrere theoretische Modelle für die Erosion einer festen Oberfläche durch einen Strahl harter Partikel, die gut mit experimentellen Ergebnissen in Einklang stehen ${ }^{2}$. Finnie [9] fasste den Verschleiß als reines Mikroschneiden auf. Beckmann und Goltzmann [10] verfeinerten diesen Ansatz, indem sie annahmen, dass für den Verschleiß neben der starken plastischen Deformation eine Scherbelastung der Oberflächenschicht vorliegen muss. Ellermaa [2] verglich verschiedene Theorien zum Schlagverschleiß mit experimentellen Versuchen und kam zu dem Schluss, dass die Vorhersagen der Theorie von Beckmann \& Goltzmann in leicht empirisch modifizierter Form - am besten mit den experimentellen Ergebnissen übereinstimmten. Molinari und Ortiz [3] führten FEM-basierte Simulationen des elastoplastischen ebenen Stoßes von Stahlkugeln auf eine weichere Stahlplatte durch, verglichen

\footnotetext{
${ }^{1}$ Teilweise sind auch die Termini „Stoßverschleiß“ oder ,,erosiver Verschleiß“ gebräuchlich. Letzterer tritt allerdings auch bei der Wechselwirkung zwischen Flüssigkeiten und festen Oberflächen auf, z. B. durch Kavitation; in dem vorliegenden Unterkapitel seien dagegen grundsätzlich trockene Bedingungen angenommen.

${ }^{2}$ Eine gute Übersicht zu theoretischen und experimentellen Untersuchungen des Schlagverschleißes metallischer Oberflächen bietet die Monografie von Kleis und Kulu [8].
} 
ihre Ergebnisse mit den Beobachtungen von Hutchings et al. [11] der entsprechenden Stoß„Krater“ und erzielten eine gute Übereinstimmung zwischen Theorie und Experiment.

Alle genannten Arbeiten beruhen (direkt oder indirekt) auf kontaktmechanisch mehr oder weniger rigorosen Beschreibungen des einzelnen Stoßproblems. Die Schwierigkeit liegt an dieser Stelle darin, dass man in der Regel das elasto-plastische Problem mit Reibung lösen muss, für das keine einfachen Modelle zur Verfügung stehen.

Grundsätzlich muss man zwischen dem Verschleiß duktiler und dem spröder Oberflächen unterscheiden. Bei spröden Körpern kommt es zu Beginn der Kollision nach der kurzen plastischen Phase zur Bildung radialer Risse, die die Festigkeit reduzieren [12]. In der Restitutionsphase bilden sich vermehrt laterale Risse, durch die das Material letztlich abgetragen wird [12]. Bei spröden Materialien tritt das Maximum der Verschleißintensität durch die Erosion bei vorgegebener Kollisionsgeschwindigkeit für den reinen Normalstoß auf, während das Maximum bei duktilen Werkstoffen in der Regel bei einem bestimmten schiefen Winkel liegt [8, S. 21].

Verbindet man das klassische Gesetz von Khrushchov und Babichev [13] für den abrasiven Verschleiß - nach dem die Verschleißintensität proportional zur Normalkraft und zur Gleitgeschwindigkeit ist - mit dem Reibgesetz von Amontons und Coulomb, ergibt sich ein energiebasiertes Verschleißgesetz, das für den abrasiven Verschleiß zuerst von Honda und Yamada [14] vorgeschlagen wurde und nach dem die Verschleißintensität proportional zur dissipierenden Reibleistung ist. Energiebasierte Verschleißgesetze sind bei der Untersuchung des Schlagverschleißes bereits erfolgreich eingesetzt worden [15, 16]; auch das Konzept der konstanten spezifischen Energie [8, S.67] steht mit dieser Idee in Einklang. Dieses bezieht sich zwar auf die Energie der Partikel vor der Kollision, ist aber im Zusammenhang mit hoch-plastischen Stößen entstanden, für die die gesamte Energie während der Kollision dissipiert.

Das Elegante der energiebasierten Betrachtung besteht darin, dass zur Bestimmung des gesamten Verschleißvolumens nach der Kollision wegen Gl. (2.56) nur die beiden Stoßzahlen bekannt sein müssen; diese können für viele Konfigurationen in den vorangegangenen Teilen diese Buches nachgeschlagen werden. Die Form des Stoß-,,Kraters“ ergibt sich in der energiebasierten Betrachtung aus der Verteilung der dissipierten Energie über das Kontaktgebiet während der Kollision. Für den elastischen Stoß mit Reibung wurde dieses Problem im Abschn. 6.3.1 bereits diskutiert.

\subsection{Stoßbasierte Testverfahren}

Rückprall- oder Fallgewichtsversuche sind einfache und schnelle Methoden, um die mechanischen Charakteristika von Probenkörpern zu ermitteln. Die zu untersuchenden Materialeigenschaften können dabei (aber müssen nicht unbedingt) mit dynamischen Lastkonfigurationen (z.B. hohen Deformationsgeschwindigkeiten) assoziert sein. Eine gute 
Übersicht zu experimentellen Verfahren für Untersuchungen mit hohen Deformationsgeschwindigkeiten bietet die Arbeit von Field et al. [17].

Außerdem kommen stoßbasierte Testverfahren zum Einsatz, wenn tatsächliche Kollisionen simuliert werden sollen, beispielsweise bei der Auslegung und Prüfung von Schutzhelmen.

\subsubsection{Materialprüfung durch Rückprallversuche}

\section{Rückprall-Elastizität von Elastomeren}

Rückpralltests verwendet man unter anderem als einfache Möglichkeit, Informationen über die rheologischen Eigenschaften von Elastomeren unter dynamischer Belastung zu erhalten. Die Stoßzahl - oder eine direkt daraus ableitbare äquivalente Größe wie die Rückprallhöhe bezeichnet man in diesem Zusammenhang als Maß der „Rückprall-Elastizität“”.

Verfahren zur Bestimmung dieser Elastomer-Eigenschaft sind in den Normen DIN EN ISO 8307 (Kugel-Rückprall-Test) und DIN 53512 (Bestimmung durch ein sogenanntes Schob-Pendel, siehe Abb. 8.1) geregelt. Außerdem beschäftigte sich schon Bassi [18] mit der Bestimmung des dynamischen Moduls eines Elastomers durch den Rückprall einer starren Kugel.

Es stellt sich die Frage, wie der einzelne im Rückpralltest bestimmte Wert - die Stoßzahl im Allgemeinen von der ganzen komplizierten Rheologie des Elastomers abhängt. Ist es, mit anderen Worten, möglich, die gemessene Stoßzahl mit einer konkreten rheologischen Information in Verbindung zu bringen ${ }^{3}$ ?

Abb. 8.1 Schematischer Aufbau eines Schob-Pendels zur Bestimmung der Rückprall-Elastizität einer Elastomerprobe (schwarz). Als $\mathrm{Maß}$ der Elastizität fungiert die Rückprallhöhe - Zustand (c) der gelenkig gelagerten Hammerfinne

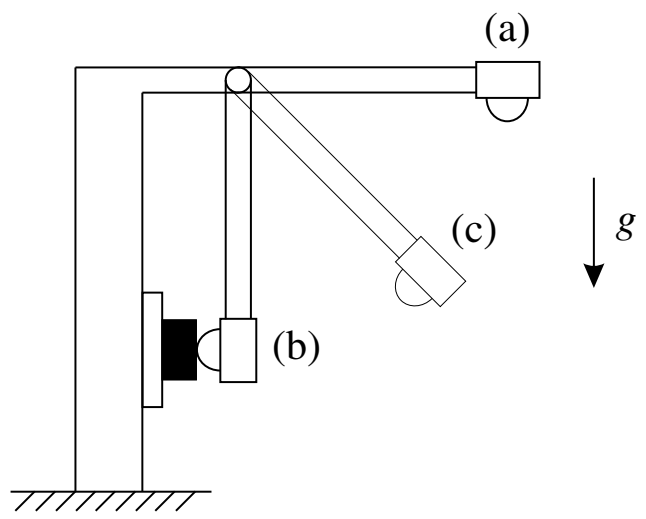

\footnotetext{
${ }^{3}$ Die Aussage „Die Stoßzahl ist ein Maß der Rückprall-Elastizität.“ ist per Definition richtig und daher tautologisch.
} 
Wie im Abschn. 5.4 gezeigt wurde, ist das tatsächlich der Fall: (Weitgehend) unabhängig von der konkreten weiteren Rheologie und dem Profil des Rückprallkörpers ist die Stoßzahl eine bestimmte Funktion des Verhältnisses zwischen Verlust- und Speichermodul bei der charakteristischen Zeitskala des Stoßes. Mithilfe der Gl.(5.56) und (5.61) lässt sich außerdem aus der Stoßdauer eine weitere Information über die beiden Moduln extrahieren. Deswegen ist durch den Rückpralltest der elastische Modul und die Viskosität des Elastomers auf der Zeitskala der Stoßdauer bestimmbar.

Man muss dabei allerdings bedenken, dass die im Abschn. 5.4 erhaltenen Ergebnisse unter vereinfachenden Annahmen zustande gekommen sind. Nicht berücksichtigt wurden beispielsweise die Wellenausbreitung (die gerade für weiche Materialien wie Elastomere sehr relevant sein kann) oder der Mullins-Effekt, also die Entfestigung einer Elastomer-Probe durch wiederholte Belastung.

\section{Härteprüfung nach Leeb}

Auch für metallische Proben kommen Rückpralltests zum Einsatz, in denen aus der Stoßzahl eines harten Rückprallkörpers auf plastische Eigenschaften - Fließgrenze oder Härte - der Probe geschlossen wird. Diese „Härteprüfung nach Leeb“ ist in den Normen DIN EN ISO 16859-1 bis DIN EN ISO 16859-3 geregelt.

Im Unterkapitel zum elasto-plastischen Normalstoß wurde dabei gezeigt, dass die Stoßzahl hauptsächlich von dem Verhältnis zwischen der Stoßgeschwindigkeit und der kritischen Geschwindigkeit - die zur Erzeugung plastischer Deformationen nötig ist - bestimmt ist. Allerdings spielt auch die Poissonzahl des indentierten Mediums eine (geringe) Rolle. Das Profil des Eindruckkörpers wurde in seinem Einfluss nicht genauer untersucht, ist aber vermutlich ebenfalls von Bedeutung, zumindest wenn der Körper scharfe Kanten aufweist, die wegen der auftretenden Spannungsspitzen das Fließverhalten verändern.

\section{Dynamische Härtemessung nach Taylor}

Taylor [19] schlug ein Verfahren zur Bestimmung der dynamischen Härte vor, das in Abb. 8.2 schematisch dargestellt ist: eine zylindrische Probe aus dem zu untersuchenden Material stößt mit relativ hoher Geschwindigkeit gegen eine starre Wand. Aus der Differenz zwischen der ursprünglichen Länge der Probe und der deformierten Länge nach der Kollision sowie der Stoßgeschwindigkeit kann man die Beschleunigung der Probe und damit die dynamische Härte abschätzen.

Abb. 8.2 Schematische Darstellung des Taylor-Tests zur Bestimmung der dynamischen Härte einer zylindrischen Probe

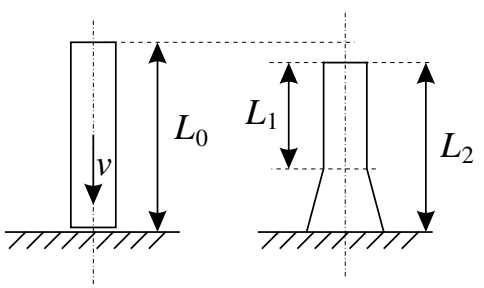


Taylor nahm an, dass sich die Probe starr-plastisch deformiert und untersuchte die Massen- und Impulserhaltung für den plastisch deformierten und den undeformierten Teil der Probe. Zur Schließung des entstehenden Gleichungssystems benötigt man allerdings eine Aussage über die Ausbreitungsgeschwindigkeit der plastischen Wellenfront. Jones et al. [20] vervollständigten diese elementare Theorie des Verfahrens durch die Annahme, dass diese Geschwindigkeit proportional zu der Geschwindigkeit des undeformierten Teils der Probe ist. Lu et al. [21] erweiterten die Theorie um die Berücksichtigung der Kompressibilität des Materials. Sarva et al. [22] führten vollständige FEM-basierte Simulationen des Tests mit einer Probe aus Polycarbonat durch.

\section{Fallgewichtstests an Gelenkknorpel}

Stoßartige Belastungen sind eine bedeutende Quelle der Schädigung von Knorpelgewebe ${ }^{4}$. Zum vertieften Verständnis der biomechanischen Reaktion des Gewebes auf die Belastung ist die Kenntnis der elastischen und viskoelastischen Eigenschaften des Gewebes eine unabdingbare Voraussetzung.

Zur Bestimmung dieser Eigenschaften kommen häufig Fallgewichtstests zum Einsatz. Die besondere Schwierigkeit besteht dabei in der empfindlichen Natur der Versuchsproben. Burgin und Aspden [23] publizierten daher einen speziellen Fallturm, um die Kontaktkräfte auf das Gewebe in dem Fallgewichtsversuch zu untersuchen. Diese Apparatur wurde später von Kang et al. [24] zur Analyse möglicher Kavitation in dem Gelenkknorpel während der Restitutionsphase weiterentwickelt. Zur theoretischen Beschreibung des Kontaktes verwendeten sie ein einfaches rheologisches Modell mit zwei Freiheitsgraden.

Ruta und Szydło [25] schlugen ein analytisches Verfahren vor, wie man aus den dynamischen Ergebnissen eines Fallgewichtstests mit einem elastischen Medium die statischen Moduln des Materials bestimmen kann. Mithilfe der dynamischen Fundamentallösung des elastischen Halbraums bestimmten sie die Verschiebungen des Mediums einer gleichförmig im kreisförmigen Kontaktgebiet verteilten harmonischen Normalkraft. Wie in den früheren Kapiteln dieses Buches dargestellt, ist die harmonische Näherung ein gutes Modell für die Normalkraft während der Kollision. Eine konstante Druckverteilung im Kontaktgebiet wird hingegen von einem sehr speziellen Eindruckkörper erzeugt (siehe [26, S. 23 f.]); ein Fallgewichtstest entspricht dagegen am ehesten einem zylindrischen Flachstempel mit der in Gl. (3.22) gegebenen Druckverteilung.

Der Normalstoß eines zylindrischen Flachstempels auf ein viskoelastisches Medium ist im fünften Kapitel dieses Buchs ausführlich dargestellt. Da der Flachstempel-Kontakt linear ist, ist die Behandlung durch ein einfaches rheologisches Modell durchaus berechtigt. Wie oben ausgeführt, hängt das Stoßverhalten linear-viskoelastischer Medien hauptsächlich von einem rheologischen Faktor ab, der das Verhältnis von Verlust- und Speichermodul bei der Zeitskala der Stoßdauer wiedergibt. Die Materialeigenschaften bei langsamer Belastung des Knorpels können sich daher wesentlich von denen in stoßbasierten Tests unterscheiden, wie Burgin et al. [27] demonstrierten.

\footnotetext{
${ }^{4}$ Auf diesen Aspekt wird im Abschn. 8.6 genauer eingegangen werden.
} 
Gelenkknorpel ist allerdings ein mehrphasiges, faserverstärktes, viskoelastisches Material. Einen großen Einfluss auf die viskoelastischen Eigenschaften von Knorpelgewebe hat beispielsweise bei langsameren Belastungen (mit charakteristischen Anregungsfrequenzen von bis zu $100 \mathrm{~Hz}$ ) dessen Hydration. Eine größere Hydration führt dabei (etwas paradoxerweise) dazu, dass das Verhältnis von Speicher- und Verlustmodul wächst [28]; das Gewebe verliert dadurch an Fähigkeit, Energie durch viskose Deformationen zu dissipieren. Bei sehr schnellen stoßartigen Belastungen ist es dagegen unwahrscheinlich, dass der Flüssigkeitsanteil (als zweite Phase) Einfluss auf die Dissipation hat [29]. In diesem Fall sind daher mehrphasige Beschreibungen des Knorpelgewebes oft gar nicht notwendig.

Selyutina et al. [30] verwendeten ein quasi-lineares Kelvin-Voigt-Modell zur Beschreibung des Fallgewichtstests mit Gelenkknorpel und verglichen ihre Vorhersagen mit experimentellen Ergebnissen. Das Modell wurde kürzlich von Springhetti und Selyutina [31] verallgemeinert, um große Deformationen des Gewebes berücksichtigen zu können.

Pierce et al. [32] schlugen schließlich ein Mikrostruktur-basiertes Kontinuumsmodell zur Beschreibung der viskoelastischen und permeablen Eigenschaften von Knorpel-Gewebe vor, das die statistische Verteilung der Faserorientierungen in dem Material in Betracht zieht.

\section{Fallgewichtstests an Straßenbelag}

Auch zur Bestimmung der elastischen Eigenschaften von Straßenbelägen verwendet man Fallgewichtstests. Das Messprinzip ist dabei aber etwas anders, als bei dem oben erwähnten Fallturm zur Untersuchung von Knorpelgewebe ${ }^{5}$ : In bestimmten Abständen von der Last, also dem fallenden Gewicht, misst man die Verschiebung der Oberfläche und schließt daraus auf die elastischen Moduln der Schichten des Straßenbelags [33] - siehe Abb. 8.3. Die Bestimmung dieser Verschiebung ist ein rein kontaktmechanisches Problem. Die Kontaktmechanik geschichteter Medien wurde zwar in dem vorliegenden Buch nicht behandelt, dazu existiert aber eine umfangreiche Literatur. In mehrerer Hinsicht sind diese Materialien außerdem mit den in diesem Buch behandelten Gradientenmedien verwandt.

Der Fallgewichtstest ist ein dynamischer Versuch. Dies wirft wiederum die Frage auf, wie man die Ergebnisse des dynamischen Experiments in Form statischer Moduln deuten kann. Diesem Problem widmeten sich Ruta et al. [34], indem sie ihr oben für homogene elastische Medien beschriebenes Verfahren zur Umrechnung der statischen und dynamischen Lösungen für geschichtete Materialien verallgemeinerten.

\subsubsection{Weitere stoßbasierte Testverfahren}

Ein weiterer Bereich von stoßbasierten Testverfahren ist die Auslegung und Prüfung von Schutzhelmen, z. B. von Motorradfahrer*innen. Die Helmprüfung ist durch die europäische

\footnotetext{
${ }^{5}$ Das wird schon aus dem englischen Begriff für das verwendete Messgerät, ,ffalling weight deflectometer", deutlich.
} 
Abb. 8.3 Schematische

Darstellung des

Fallgewichtstests zur

Bestimmung der elastischen

Eigenschaften eines

dreischichtigen Straßenbelags

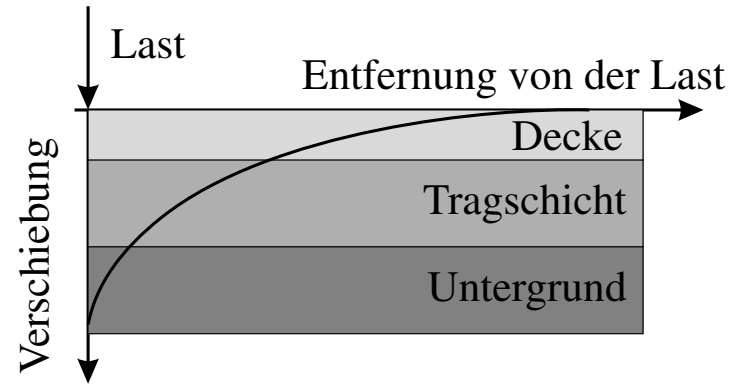

Norm ECE 22.05 reglementiert und umfasst unter anderem Stoßdämpfungswerte an einzelnen Punkten des Helms durch den Falltest.

Zur numerischen Simulation dieser Tests verwendet man in der Regel FEM-basierte Modelle [35]. Dabei hat sich herausgestellt, dass vor allem die bei einem hohen Reibungskoeffizienten zwischen Helm und Gegenkörper (Straße oder Fahrzeugkarosserie) auftretenden großen Winkelbeschleunigungen - die man schon mithilfe des in Abb.2.2 gezeigten elementaren Modells verstehen kann - potentiell gefährlich sind [36].

\subsection{Granulare Medien}

Sand, Salz, Getreide, Bergbauprodukte - Granulare Medien sind aus unserer Umgebung kaum wegzudenken. Wegen der zahlreichen einzigartigen Eigenschaften granularer Materie prägten Jaeger et al. in ihrer klassischen Arbeit [37] den Begriff des ,,zusätzlichen Aggregatzustands“. Während diese Bezeichnung aus thermodynamischer Sicht natürlich fragwürdig ist - granulare Medien bestehen aus einer Vielzahl einzelner Festkörper-Körner - gibt es tatsächlich mehrere Dinge, in denen sich granulare Materie von „normalen“ Fluiden oder Festkörpern unterscheidet. Je nach der Belastung kann sie sich wie ein Gas, eine Flüssigkeit (wie in einer Sanduhr) oder ein geordneter Festkörper verhalten ${ }^{6}$. In jedem Fall ist das Verhalten dabei besonders.

\subsubsection{Kinetische Theorie granularer Medien}

Die kinetische Beschreibung von granularen Medien gehört zu den frühesten Versuchen, das Verhalten dieser Materialien systematisch zu analysieren. Lun und Savage [39] publizierten eine einfache kinetische Theorie für den Fall, dass sich das granulare Medium durch inelastische raue Kugeln konstituiert und die mittlere freie Weglänge zwischen den Kugeln sehr viel

\footnotetext{
${ }^{6}$ Eine aktuelle und systematische Darstellung zu den „Aggregatzuständen“ granularer Materie bietet die Monografie von Andreotti et al. [38].
} 
größer ist als der mittlere Kugelradius ${ }^{7}$. Eine sehr gute Zusammenfassung der zahlreichen Arbeiten aus den 80-er Jahren zur kinetischen und kontinuumstheoretischen Beschreibung granularer Materie bietet die Publikation von Campbell [40].

Die Grundlage dieser Theorien bildet die Boltzmann-Gleichung für die EinteilchenVerteilungsfunktion $p(\underline{r}, \underline{v}, \underline{s}, t)$ (mit dem Ortsvektor $\underline{r}$, der Geschwindigkeit $\underline{v}$ und dem Spin $\underline{s}$ ) eines Partikels des granularen Mediums. Unter der Annahme der Abwesenheit äußerer Kräfte nimmt die Boltzmann-Gleichung die Form

$$
\frac{\partial p}{\partial t}+\underline{v} \cdot \nabla p=\left(\frac{\partial p}{\partial t}\right)_{S}
$$

an. Die rechte Seite dieser Gleichung bezeichnet die Änderung der Verteilungsfunktion durch Stöße von Teilchen. Diese Änderung besteht aus einer Verlustrate durch Teilchen, die im Zuge einer Kollision aus dem Zustand $(\underline{v}, \underline{s})$ herausgestreut werden, und einer Gewinnrate durch Teilchen mit dem Zustand $\left(\underline{v}^{*}, \underline{s}^{*}\right)$ vor der Kollision, die nach einer Kollision den Zustand $(\underline{v}, \underline{s})$ aufweisen. Unter den Annahmen, dass alle Teilchen den Radius $R$ haben und die Verteilungsfunktionen zweier Teilchen unabhängig voneinander sind, lautet der Stoßoperator dann wie folgt [41, 42, S.32]:

$$
\begin{aligned}
& \left(\frac{\partial p\left(\underline{r}, \underline{v}_{1}, \underline{s}_{1}, t\right)}{\partial t}\right)_{S}=(2 R)^{2} \iiint \mathrm{H}\left(-v_{z, K}\right)\left|v_{z, K}\right| \\
& \times\left(\frac{1}{\epsilon_{z} D} p\left(\underline{r}, \underline{v}_{1}^{*}, \underline{s}_{1}^{*}, t\right) p\left(\underline{r}, \underline{v}_{2}^{*}, \underline{s}_{2}^{*}, t\right)-p\left(\underline{r}, \underline{v}_{1}, \underline{s}_{1}, t\right) p\left(\underline{r}, \underline{v}_{2}, \underline{s}_{2}, t\right)\right) \mathrm{d} \underline{e}_{z} \mathrm{~d} \underline{v}_{2} \mathrm{~d} \underline{s}_{2} .
\end{aligned}
$$

Die Heaviside-Funktion H sorgt dafür, dass nur Zustandspaare betrachtet werden, für die tatsächlich eine Kollision stattfindet. $D$ ist die Jacobi-Determinante der in den Gl. (2.45)-(2.48) gegebenen Transformation, mit der die Geschwindigkeiten und Spins nach der Kollision aus denen vor dem Zusammenstoß bestimmbar sind.

Ohne an dieser Stelle genauer auf die Bestimmung von $D$ einzugehen, ist klar, dass $D$ sich im Fall konstanter Stoßzahlen elementar durch diese Stoßzahlen ausdrücken lässt. Tatsächlich sind die Stoßzahlen aber, wie in früheren Kapiteln gesehen, grundsätzlich und nicht-trivial von den Stoßgeschwindigkeiten selbst abhängig, egal, ob man Viskoelastizität, Plastizität, Adhäsion, Reibung oder eine Kombination dieser Phänomene als grundlegenden Dissipationsmechanismus bei der Kollision betrachtet. Die Bestimmung der JacobiDeterminante und damit die ganze analytische Entwicklung einer kinetischen Theorie granularer Medien wird deswegen durch die Geschwindigkeitsabhängigkeit der Stoßzahlen massiv erschwert.

\footnotetext{
${ }^{7}$ Man spricht dann von einem „Granularen Gas“ (siehe weiter unten).
} 
Die meisten Arbeiten auf diesem Gebiet arbeiten daher unter der (aus kontaktmechanischer Sicht eigentlich unsinnigen) Annahme konstanter Stoßzahlen. Die Geschwindigkeitsabhängigkeit sorgt aber bei der Dynamik granularer Medien für mehrere qualitativ neue Effekte, wie in Abschn. 8.3.3 dargelegt wird. Die ersten Versuche, diese Abhängigkeiten in die kinetische Theorie zu integrieren, stammen von Walton und Braun [43] sowie Lun und Savage [44]. Brilliantov und Pöschel [45] gaben eine Reihenentwicklung der JacobiDeterminante für den Fall glatter viskoelastischer Kugeln im Rahmen des im Abschn. 5.4 diskutierten Kuwabara-Kono-Modells an und untersuchten den resultierenden Abkühlungsprozess des granularen Gases.

Die Geschwindigkeitsabhängigkeit der Stoßzahlen berücksichtigen Forscher*innen inzwischen allerdings häufig im Rahmen der numerischen Simulation der Dynamik granularer Medien ${ }^{8}$, auf die im folgenden Abschnitt kurz eingegangen wird.

\subsubsection{Numerische Simulation granularer Medien}

Die kinetische und hydrodynamische Beschreibung granularer Medien beruht auf Annahmen, deren Erfüllung durch reale granulare Materialien alles andere als selbstverständlich ist. Beispielsweise verletzen dissipative Gase die Annahme von molekularem Chaos [48], d.h. die Verteilungsfunktionen der Zustände zweier Partikel des granularen Mediums sind nicht unabhängig voneinander.

In experimentellen Untersuchungen sind wiederum wesentliche Größen, wie die Positionen und Geschwindigkeiten einzelner Partikel, einer direkten Messung häufig unzugänglich.

Für eine umfassende und robuste Beschreibung der Dynamik granularer Medien sind daher numerische Simulationen unabdingbar (und sehr weit verbreitet), wobei in der Regel die Diskrete-Elemente-Methode (DEM) ${ }^{9}$ zum Einsatz kommt. Zur numerischen Simulation der Dynamik granularer Medien gibt es eine sehr umfangreiche Literatur (siehe beispielsweise die Monografien von Pöschel und Schwager [49] und Zohdi [50]), außerdem stehen für dieses Problem flexible Software-Pakete wie LAMMPS zur Verfügung. Im Folgenden soll daher nur auf einige Punkte näher eingegangen werden, für die die in den vorherigen Kapiteln dieses Buches erhaltenen Ergebnisse von Bedeutung sein können.

\section{Zeitgesteuerte DEM}

Der in gewisser Weise einfachste Ansatz zur numerischen Simulation der Dynamik granularer Materie besteht sicher darin, die Dynamik jedes einzelnen Partikels (Elements) direkt zu simulieren. Das ist die Idee der zeitgesteuerten DEM. Die Partikel behalten während der

\footnotetext{
${ }^{8}$ siehe beispielsweise die Arbeiten von Schwager und Pöschel [46] sowie Dubey et al. [47].

${ }^{9}$ Wegen ihrer sehr engen Verwandtschaft mit der Molekulardynamik wurde die DEM früher häufig unter deren Namen subsumiert; die simulierten Partikel sind in der DEM aber weder molekularer noch quasi-molekularer sondern makroskopischer Natur.
} 
Simulation ihre makroskopische Form (abgesehen von Fällen, in denen ein Auseinanderbrechen oder „Verklumpen“ der Teilchen berücksichtigt werden soll), um die Bewegungsgleichungen, also die Impuls- und Drehimpulssätze für jedes Partikel, möglichst einfach zu halten. Häufig nimmt man an, dass die Elemente - bis auf Randbedingungen - nur untereinander wechselwirken, die Kraft auf das Element mit dem Index $i$ ergibt sich dann beispielsweise durch die Summe der Wechselwirkungen mit allen anderen Elementen,

$$
\underline{F}_{i}=\sum_{j \neq i} \underline{F}_{i j}
$$

Die Aufgabe besteht nun aus zwei Teilen: der Bestimmung der Wechselwirkungen und der Integration der Bewegungsgleichungen (beide Teile können in hohem Maße parallelisiert werden). Abgesehen von dem algorithmischen Problem, möglichst effizient festzustellen, welche Elemente überhaupt miteinander wechselwirken, ist der erste Teil eine kontaktmechanische Frage. Leider haben wir gesehen, dass die Angabe eines expliziten Kraftgesetzes für die Kontaktkräfte als Funktionen der Positionen und Geschwindigkeiten der Kontaktpartner problematisch ist, wenn die Kontaktkonfiguration - durch inelastische Deformationen oder Reibung - von der Belastungsgeschichte des Kontaktes abhängt. Häufig behilft man sich in solchen Fällen mit expliziten aber dafür semi-rigorosen Kraftgesetzen ${ }^{10}$. Eine rigorose Variante wären hybride Modelle, bei denen ein Kontaktprogramm, beispielsweise auf der Grundlage der MDR, in die DEM-Simulation integriert wird. Das wäre zwar - im Vergleich zur Verwendung expliziter Kraftgesetze - nicht sehr effizient, aber angesichts der Rechenleistung moderner Computer durchaus denkbar.

\section{Ereignisgesteuerte DEM}

Wenn die charakteristische Stoßdauer zwischen zwei Partikeln sehr klein gegenüber der mittleren Zeit ist, die sich ein Element frei in dem granularen Medium bewegen kann, und die Wechselwirkungen daher in überwältigender Mehrheit aus binären Kollisionen bestehen (z.B. bei granularen Gasen), ist es nicht nötig, die Bewegungsgleichungen für jedes Element zu formulieren und in der Zeit zu integrieren. Stattdessen kann man die Dynamik des granularen Mediums als Folge instantaner Kollisionen (Ereignisse) auffassen, die durch die Angabe der (geschwindigkeitsabhängigen) Stoßzahlen vollständig beschreibbar sind. Das algorithmische Problem besteht dann „nur“ darin, die einzelnen Ereignisse zu planen und auszuführen; für diese Aufgabe publizierte Lubachevsky [52] eine sehr effiziente Lösung.

Dieses Verfahren ist ebenfalls in hohem Maße parallelisierbar (beispielsweise durch die Unterteilung des Simulationsraums in kleinere Einheiten) und arbeitet deutlich schneller als die zeitgesteuerte DEM. Außerdem wird das geschilderte Problem der Angabe von expliziten Kraftgesetzen für die Kontaktkräfte umgangen, da die Stoßzahlen (wie in dem vorliegenden Buch ausführlich dargestellt) auch in inelastischen Kollisionen oder solchen mit Reibung in allgemeiner Form bestimmbar sind.

\footnotetext{
${ }^{10}$ Im Fall des viskoelastischen Normalkontaktes verwendet man beispielsweise oft das KuwabaraKono-Modell, siehe auch die vergleichende Studie von Kačianauskas et al. [51].
} 


\section{Weitere numerische Verfahren}

Neben der DEM kommen teilweise auch andere numerische Verfahren zum Einsatz, um die Dynamik granularer Medien zu untersuchen.

In Monte-Carlo-basierten Methoden (direct simulation Monte Carlo, DSMC) werden nicht mehr die Trajektorien der einzelnen Teilchen des Materials bestimmt, sondern die Entwicklung der Wahrscheinlichkeitsdichte für einen bestimmten Zustand des Gesamtsystems. Mathematisch läuft das auf die Lösung der Boltzmann-Gleichung (8.1) hinaus ${ }^{11}$. Dies ist in der Regel mit weiteren vereinfachenden Annahmen (siehe Abschn. 8.3.1), aber dafür auch mit einer deutlichen Effizienz-Steigerung gegenüber der DEM verbunden.

Außerdem setzt man sehr vereinzelt Mehrkörpersimulationen (MKS) und zelluläre Automaten zur Analyse ein.

\subsubsection{Formen granularer Medien}

\section{Granulare Gase}

Eine Portion granularer Materie kann durch ausreichend starke Anregung (beispielsweise mithilfe von Vibrationen, Gravitation oder Scherung) in einen Zustand gebracht werden, in dem die mittlere freie Weglänge so groß ist, dass die Teilchen nur durch einzelne, binäre Kollisionen wechselwirken. Diesen Zustand granularer Materie bezeichnet man als „Granulares $\mathrm{Gas}^{* 12}$.

Die Ähnlichkeit zu molekularen Gasen - die sich auch in der frühen theoretischen Beschreibung granularer Gase widerspiegelt (siehe den obigen Abschn. 8.3.1) - liegt auf der Hand. Allerdings gibt es in granularen Gasen mehrere einzigartige Phänomene, die vor allem auf die drei folgenden fundamentalen Unterschiede zu molekularen Gasen zurückzuführen sind:

- Die einzelnen Kollisionen in granularen Gasen sind auf komplexe Art und Weise und grundsätzlich mit Energiedissipation verbunden. Ein granulares Gas nichtverschwindender Gesamtenergie besitzt deswegen niemals ein thermodynamisches Gleichgewicht.

- Die Partikel, die das granulare Gas konstituieren, haben makroskopische Ausmaße und die Teilchenzahl in einem granularen System liegt mehrere Größenordnungen unter der Avogadro-Zahl. Einerseits wird dadurch die eventuelle Brownsche Bewegung der Partikel irrelevant, andererseits können Effekte, die in molekularen System nach der Mittelung über das statistische Ensemble verschwindend klein sind, in granularen Systemen durchaus eine Rolle spielen.

\footnotetext{
${ }^{11}$ Monte-Carlo-Simulationen werden daher häufig verwendet, um analytische Ergebnisse der kinetischen Theorie granularer Medien zu bestätigen.

${ }^{12}$ Im englischen Sprachraum war früher auch die Bezeichnung , rapid granular flow“ verbreitet.
} 
- Außerdem gibt es in granularen Gasen wegen des makroskopischen Charakters der Teilchen keine klare Skalentrennung zwischen der charakteristischen Länge eines einzelnen Teilchens, mesoskopischen Längen „,dx“, auf denen Gradienten kontinuierlicher (d.h. gemittelter) Größen definiert werden können, und der makroskopischen Länge des Gesamtsystems.

Insbesondere aufgrund des letzten Punktes ist fraglich, ob granulare Gase überhaupt auf der Grundlage kontinuumstheoretischer Modelle (beispielsweise hydrodynamischer Gleichungen) adäquat beschreibbar sind [53].

Da granulare Gase keinen Gleichgewichtszustand mit nicht-verschwindender Gesamtenergie besitzen, behilft man sich - wenn in der Thermodynamik des Nichtgleichgewichts der Gleichgewichtszustand, zu dem das System konvergiert, gebraucht wird, z. B. für Reihenentwicklungen in der Nähe des Gleichgewichts - in der Thermodynamik granularer Gase mit einem zuerst von Haff [54] betrachteten quasi-Gleichgewicht, in dem alle statistischen Größen homogen und isotrop verteilt sind und in dem das Gas durch die Energiedissipation in den Kollisionen kontinuierlich abkühlt.

Dieser in der englischen Literatur als „,homogeneous cooling state“ (HCS) bezeichnete Zustand ist allerdings instabil, wie man sich durch ein einfaches thermodynamisches Argument klarmacht: Kommt es durch statistische Fluktuationen in einer Region des granularen Gases zu einer lokal erhöhten Teilchendichte, finden in dieser Region vermehrt Kollisionen statt. Durch die deswegen lokal erhöhte Energiedissipation sinkt der Druck und es werden weitere Teilchen in die Region gezogen. Das granulare Gas bildet daher Cluster [55].

Interessanterweise hängt diese Cluster-Bildung sehr stark von der Geschwindigkeitsabhängigkeit der Stoßzahlen ab. Kühlt das Gas an einem Ort durch die lokal erhöhte Teilchendichte schneller ab, wird die mittlere Geschwindigkeit der Teilchen in dieser Region kleiner. Wenn die Energiedissipation hauptsächlich aus der Inelastizität der Kollision stammt, steigt allerdings die Stoßzahl in der Regel bei fallender Geschwindigkeit, was die weitere Abkühlung und Cluster-Bildung bremst. Falls wiederum der Energieverlust während der Kollision hauptsächlich durch adhäsive Beiträge zustande kommt, sinkt die Stoßzahl mit kleiner werdender Geschwindigkeit und die Cluster-Bildung wird beschleunigt. Die langfristige Dynamik großer granularer Systeme unter der Berücksichtigung der Geschwindigkeitsabhängigkeit der Stoßzahlen ist daher ein interessantes und noch weitgehend offenes Forschungsproblem.

\section{Dichte granulare Packungen}

Wenn die mittlere freie Weglänge in einem granularen Medium sehr viel kleiner als der charakteristische Durchmesser der Partikel wird, geht das Material in einen gepackten ,festen“ Zustand über. Den Übergang vom granularen Gas zur granularen Packung dokumentierten beispielsweise Falcon et al. [56] experimentell für den Fall von durch Vibrationen angeregten Stahl-Kügelchen. 
Das Verhalten granularer Packungen ist ein komplexer Forschungszweig an der Schnittstelle von Bodenmechanik, Statistischer Physik und (in deutlich weniger präsentem Maß) Kontaktmechanik. Jede dieser Disziplinen hat einen eigenen Zugang zu dem Thema ${ }^{13}$, was eine einheitliche Darstellung schwierig macht.

Im statischen Fall bilden die Kontakte zwischen den einzelnen Partikeln des granularen Mediums ein Netzwerk von Kräften, das in aller Regel statisch unbestimmt ist. Wegen dieser Unbestimmtheit ist es eigentlich notwendig, die elastische (oder auch inelastische) Wechselwirkung im Kontakt korrekt zu berücksichtigen - und zwar sowohl für den Normalkontakt als auch, wegen der Reibung im Kontakt, für den Tangentialkontakt. Dies geschieht allerdings häufig nicht [58].

Durch Mittelung des Kontaktkräftenetzwerkes über einen bestimmten Bereich kann man den lokalen Spannungszustand definieren. Dichte granulare Packungen weisen dabei elastisches und plastisches Verhalten auf. Die elastische Reaktion ist stark nicht-linear, da die Steifigkeit der Packung mit zunehmender Kompression wächst. Der Spannungszustand ist darüber hinaus in der Regel stark inhomogen. Eine nicht ganz aktuelle aber dafür genaue Übersicht zur Literatur über Spannungen in granularen Packungen liefert das Review von Savage [59]. Im Fall starr-plastischer Deformation und unter der Annahme, dass im statischen Zustand alle Kontakte den Grenzfall der Haftbedingung erfüllen, erhält man das klassische Mohr-Coulombsche Bruchkriterium

$$
\sigma_{S}=\sigma_{N} \tan \phi
$$

beziehungsweise unter Berücksichtigung der Adhäsion zwischen den Partikeln

$$
\sigma_{S}=\sigma_{0}+\sigma_{N} \tan \phi
$$

Hier bezeichnen $\sigma_{S}$ und $\sigma_{N}$ die Scher- und Normalspannung in dem versagenden Querschnitt der Packung, $\phi$ ist der Winkel der inneren Reibung und $\sigma_{0}$ der Beitrag aus der adhäsiven Wechselwirkung.

\section{Granulare Ketten}

Eindimensionale granulare Medien, oder granulare Ketten, werden häufig als Modellsysteme analysiert, um den mathematischen und numerischen Aufwand der Untersuchung zu reduzieren. Da mehrere der einzigartigen Eigenschaften granularer Materie hauptsächlich auf die Eigentümlichkeiten der Kontaktwechselwirkung zurückzuführen sind, kann man durch die Untersuchung eines eindimensionalen Modells oft zumindest ein qualitatives Verständnis der auftretenden Effekte erreichen.

In der klassischen Arbeit [60] untersuchte Nesterenko die quasistatische Ausbreitung von Störungen in einer dichten (vorgespannten) Kette elastischer Kugeln als ein Beispiel eines nichtlinearen dynamischen Systems. Wegen der starken Nichtlinearität des elastischen

\footnotetext{
${ }^{13}$ Einen guten Überblick zu den Herangehensweisen aus dem Bereich der Bodenmechanik bietet beispielsweise die Artikelsammlung [57].
} 
(Hertzschen) Kontaktes breiten sich die Störungen nicht in der Form harmonischer Wellen, sondern als Solitonen ${ }^{14}$ aus, da es für benachbarte Kugeln energetisch nicht sinnvoll ist, über längere Zeiträume relativ zueinander in gestörter Position zu verharren [61]. Diese Solitonen treten nicht nur in (Hertzschen) granularen Ketten auf ${ }^{15}$ und waren in den letzten 20 Jahren Gegenstand intensiver Forschung; untersucht wurde beispielsweise das Reflexionsverhalten an den Kettenenden [63], die Beeinflussung der Störungsausbreitung durch die Vorspannung [64] und der Einfluss der Plastizität [65]. Die genaue Form der Wechselwirkung in den einzelnen Kontakten beeinflusst dabei maßgeblich die Eigenschaften und die Ausbreitung der Solitonen.

Ein weiteres interessantes Problem, das direkt mit den Ergebnissen der vergangenen Kapitel dieses Buches verknüpft ist, ist der Energietransfer durch eine granulare Kette von $N+1$ freien (harten) Kugeln. Die Kugeln seien dabei nicht von vornherein in direktem Kontakt und wechselwirken daher nur durch binäre Stöße. Der Abstand zwischen den Kugeln sei dabei so groß, dass nur die erste Kollision zwischen zwei benachbarten Kugeln für den Energietransport relevant ist; die Masse der Kugeln sei $m_{k}$, wobei $k=0,1,2, \ldots$ den Index der Kugel in der Kette bezeichnet. Die erste Kugel habe die Geschwindigkeit $v_{0}$, alle anderen Kettenglieder ruhen. Das Glied mit dem Index $k+1$ hat nach der Kollision mit der Kugel $k$ wegen Gl.(2.49) die Geschwindigkeit

$$
v_{k+1}=\frac{m_{k}}{m_{k}+m_{k+1}}\left[1+\epsilon\left(v_{k}\right)\right] v_{k},
$$

mit der, im allgemeinen geschwindigkeitsabhängigen, Stoßzahl $\epsilon$. Man kann nun fragen, wie die Massen $m_{k}$ verteilt sein müssen, damit eine möglichst große Energiemenge durch die Kette transportiert werden kann. Falls die Stoßzahl konstant ist, führt die Bedingung für ein Maximum von $v_{N}$ auf die Relation

$$
\frac{m_{k-1}}{m_{k}}=\frac{m_{k}}{m_{k+1}}
$$

beziehungsweise (falls das Verhältnis $m_{N} / m_{0}$ vorgegeben ist)

$$
m_{k}=m_{0}\left(\frac{m_{N}}{m_{0}}\right)^{k / N} .
$$

Abb. 8.4 zeigt die Energieeffizienz

$$
\frac{U_{N}}{U_{0}}=\frac{m_{N} v_{N}^{2}}{m_{0} v_{0}^{2}}=\frac{m_{N}}{m_{0}}\left[\frac{1+\epsilon}{1+\left(m_{N} / m_{0}\right)^{1 / N}}\right]^{N}
$$

als Funktion der Kettenlänge für verschiedene Verhältnisse $m_{N} / m_{0}$ und $\epsilon=0,9$. Die Energieeffizienz ist bei einer konstanten Stoßzahl invariant gegenüber einer Inversion des

\footnotetext{
${ }^{14}$ Das sind einzelne lokalisierte Wellenpakete.

${ }^{15}$ siehe die Übersicht von Sen et al. [62].
} 
Abb. 8.4 Energieeffizienz einer losen granularen Kette inelastischer Kugeln als Funktion der Kettenlänge für verschiedene Werte des Massenverhältnisses $m_{N} / m_{0}$ und eine Stoßzahl $\epsilon=0$, 9. Die dünne Linie bezeichnet die Asymptote aus Gl. (8.10)

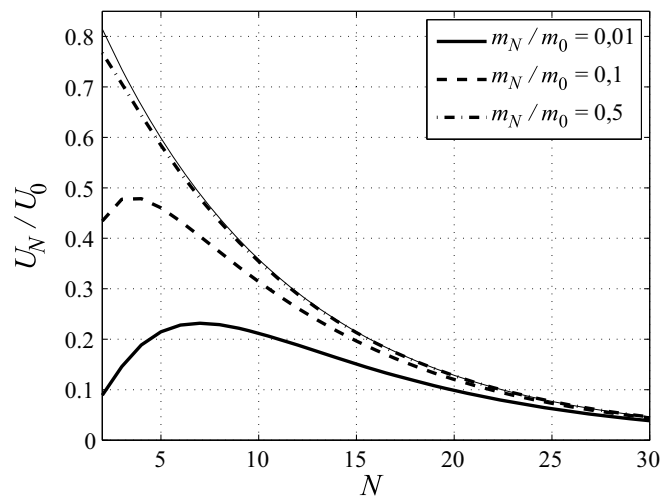

Massenverhältnisses, der Energietransport funktioniert also in beide Richtungen der Kette gleich. Für sehr große $N$ ergibt sich daher die Asymptote

$$
\lim _{N \rightarrow \infty}\left(\frac{U_{N}}{U_{0}}\right)=\left(\frac{1+\epsilon}{2}\right)^{N} .
$$

Die Annahme einer konstanten Stoßzahl ist, wie oft genug in diesem Buch dargelegt, aus kontaktmechanischer Sicht wenig sinnvoll. Je nach der Art der Inelastizität (Plastizität, Viskoelastizität o. Ä.), sieht die optimale Massenverteilung der Kette daher unterschiedlich aus. Pöschel und Brilliantov [66] untersuchten den Fall viskoelastischer Kugeln im Rahmen des Kuwabara-Kono-Modells und stellten unter anderem fest, dass die optimale Massenverteilung in diesem Fall nicht monoton ist, sondern ein Maximum aufweist, da die Stoßzahl mit fallender Geschwindigkeit wächst.

\subsection{Astrophysikalische Anwendungen}

Ein sehr populäres Beispiel dynamischer granularer Medien sind die Ringsysteme der großen Gasplaneten, insbesondere des Saturn. Diese sind zum einen an sich ein interessantes astrophysikalisches Phänomen, zum anderen erhoffen sich Wissenschaftler*innen durch das Studium dieser Ringstrukturen Erkenntnisse über die Entwicklung unseres Sonnensystems in seiner Frühphase - als es selbst eine granulare, um einen massereichen Zentralkörper rotierende Scheibe war - insbesondere über die Planetenentstehung.

Die Saturnringe bestehen aus hauptsächlich Eis- und seltener Gesteinsteilchen, deren charakteristische Längen zwischen mehreren Mikrometern und einigen Metern betragen. Die Dynamik dieser Partikel ergibt sich aus dem Zusammenspiel der inelastischen Kollisionen und der Eigengravitation zwischen den Teilchen sowie der Gravitation des massereichen Zentralkörpers. 


\subsubsection{Kollisionsmodelle für Eispartikel}

Eine Grundlage der Dynamik planetarer Ringe sind die Stöße zwischen den einzelnen Teilchen des Ringsystems. Der Zusammenhang zwischen den Stoßzahlen und den Kollisionsgeschwindigkeiten bestimmt dabei maßgeblich die Stabilität des Ringsystems, seine optische Dichte und seine Dicke [67].

Wegen der makroskopischen Gegebenheiten sind die relativen Geschwindigkeiten in diesen Kollisionen klein, höchstens wenige m/s und teilweise noch deutlich geringer [67]. In diesem Zusammenhang wurden daher in den 1980-er und 90-er Jahren, nach den Detailaufnahmen der Saturnringe durch die Raumsonde ,,Voyager 2“ im Jahr 1981, mehrere Versuche zu langsamen Kollisionen von Eiskugeln durchgeführt.

Bridges et al. [67] untersuchten Stöße von Eiskugeln gegen einen festen Eisblock mit Geschwindigkeiten zwischen 0,015 und $2 \mathrm{~cm} / \mathrm{s}$. Für die Stoßzahl $\epsilon$ als Funktion der Kollisionsgeschwindigkeit $v$ bestimmten sie den Zusammenhang

$$
\epsilon(v) \approx 0,32 v^{-0,234}
$$

wobei die Geschwindigkeit in cm/s anzugeben ist. Hatzes et al. [68] verbesserten später die verwendete experimentelle Apparatur und erhielten für Kugeln mit dem Radius $5 \mathrm{~cm}$

$$
\epsilon(v) \approx 0,94 \exp (-0,37 v)+0,002 v^{-0,85} .
$$

Die Frostablagerungen an der Oberfläche der Eiskugeln beeinflussen die Stoßzahl sehr stark. Für sehr glatte Kugeln (ohne Ablagerungen) bestimmten die Autor*innen den Zusammenhang

$$
\epsilon(v) \approx 0,82 v^{-0,047}
$$

In der späteren Arbeit [69] untersuchten Hatzes et al. außerdem den Einfluss der Frostschicht auf die kohäsive Wechselwirkung während der Kollision.

Higa et al. [70] studierten Kollisionen von Eiskugeln gegen einen Eisblock in einem größeren Geschwindigkeitsbereich, zwischen 1 und $700 \mathrm{~cm} / \mathrm{s}$. Sie erhielten für die Stoßzahl als Funktion der Stoßgeschwindigkeit den Ausdruck

$$
\epsilon(v) \approx \begin{cases}0,9 & v \leq v_{c}, \\ \left(\frac{v}{v_{c}}\right)^{-\log \left(v / v_{c}\right)}, & v>v_{c},\end{cases}
$$

mit einer temperaturabhängigen kritischen Geschwindigkeit $v_{c}$, die einen annähernd elastischen Bereich $v<v_{c}$ von dem Bereich inelastischer Kollisionen, $v>v_{c}$, trennt.

Zur Reproduktion dieser experimentellen Ergebnisse wurden einige theoretische Modelle vorgeschlagen, die auf unterschiedlichen Dissipationsmechanismen beruhen. Gorkavy und Fridman [71] entwickelten in ihrer Übersichtspublikation zur Physik der Saturnringe ein 
Abb. 8.5 Stoßzahl als

Funktion der

Kollisionsgeschwindigkeit für den Stoß einer Eiskugel auf einen Eisblock. Experimentelle Ergebnisse von Hatzes et al. [68] und verschiedene theoretische Vorhersagen

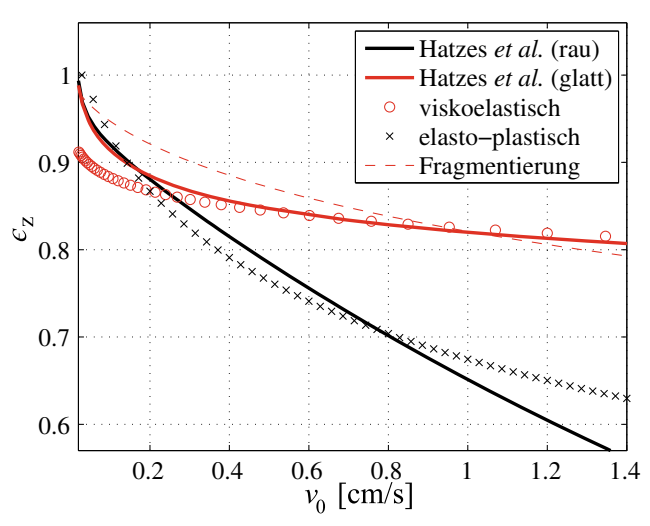

Fragmentierungsmodell, das der Physik des Energieverlustes wahrscheinlich am nächsten kommt. Für glatte Kugeln ergibt sich demnach die Stoßzahl als Lösung der Gleichung

$$
\epsilon^{2}+\left(\frac{v}{v_{0}}\right)^{2 / 3} \epsilon^{8 / 3}-1=0
$$

Die Forschergruppe um Brilliantov und Spahn [72, 73] schlug die Verwendung eines viskoelastischen Modells mit Reibung vor, das bereits im fünften und sechsten Kapitel des vorliegenden Buches diskutiert wurde. Außerdem kommen unter Umständen auch elastoplastische Ansätze zur Modellierung in Frage.

Abb. 8.5 zeigt einen Vergleich der experimentellen Kurven von Hatzes et al. [68] für raue und glatte Eiskugeln mit verschiedenen theoretischen Vorhersagen. Zur Erstellung der viskoelastischen Kurve wurde der Stoß einer Kugel auf ein inkompressibles Kelvin-Voigt-Medium mit dem elastischen Modul $G$ und der Viskosität $\eta$ rigoros gelöst (siehe Abschn. 5.4.1). Zur Skalierung der Stoßgeschwindigkeit auf dimensionsbehaftete Werte wurden die in Gl. (5.69) auftretenden Größen so gewählt, dass

$$
\frac{\tilde{m}^{2} G^{3}}{\tilde{R} \eta^{5}}=1 \times 10^{4} \mathrm{~m} / \mathrm{s} .
$$

Die Skalierung der elasto-plastischen Kurve (beruhend auf einem FEM-basierten Modell mit $v=0,3$, siehe Abschn. 5.5.1) erfolgte durch die Wahl von $v_{Y}=1 / 35 \mathrm{~cm} / \mathrm{s}$ für die kritische Geschwindigkeit, um plastische Deformationen zu erzeugen, und für die in Gl. (8.15) auftretende Normierungsgeschwindigkeit $v_{0}$ wurde der Wert $(5 / 4)^{4} \mathrm{~cm} / \mathrm{s}_{\text {angenommen }}{ }^{16}$.

\footnotetext{
${ }^{16}$ Die aufgeführten Parameter wurden grob an die Messergebnisse angepasst, aber nicht gezielt optimiert; es ist daher möglich, dass durch eine vollständige Optimierung eine bessere Übereinstimmung mit den Experimenten erzielbar ist.
} 
Man erkennt, dass das viskoelastische Modell und der Fragmentierungsansatz recht gut mit den Messergebnissen für glatte Eiskugeln in Einklang gebracht werden können. Allerdings besteht an dieser Stelle mit Sicherheit noch Bedarf nach präziseren theoretischen Modellen.

\subsubsection{Dynamik der Ringsysteme}

Wie bei anderen granularen Medien auch, verwendeten die frühen Versuche, die Dynamik planetarer Ringsysteme theoretisch zu beschreiben, kinetische und hydrodynamische Modelle. Aus hydrodynamischer Sicht führt die Energiedissipation in den einzelnen Kollisionen makroskopisch zu Reibung, bzw. zu einer Viskosität des Mediums. Da die Rotationsgeschwindigkeit wegen der Gravitation des Zentralkörpers nach außen abnimmt, führt diese viskose Scherung zu einem Transport von Drehimpuls nach außen [74].

Goldreich und Tremaine [75] bestimmten den Zusammenhang zwischen der Stoßzahl und der optischen Dichte für eine differentiell rotierende Scheibe von inelastisch kollidierenden Teilchen. Sie vernachlässigten die Eigengravitation der Partikel, nahmen an, dass die Geschwindigkeitsstreuungen normalverteilt sind, und stellten unter diesen Annahmen mithilfe der kinetischen Theorie fest, dass die optische Dichte im Allgemeinen mit der Stoßzahl wächst.

Borderies et al. [76] untersuchten die Ausbreitung und Dämpfung von Dichte-Wellen in einer rotierenden granularen Scheibe im Rahmen einer hydrodynamischen Beschreibung.

Bereits aus der kinetischen Beschreibung geht dabei hervor, dass planetare Ringsysteme anfällig für verschiedene radiale Instabilitäten sind [71] und deswegen in eine Vielzahl einzelner dünner Ringe zerfallen [77].

Diese Instabilitäten wurden seit den 1990-er Jahren auch verstärkt durch DEM-basierte numerische Simulationen genauer untersucht. Ein zentraler Baustein dieser Simulationen ist dabei die angemessene kontaktmechanische Modellierung des einzelnen Stoßprozesses.

Salo [78] untersuchte die Ausbildung von wirbelhaften Verdichtungen (sogenannten „wakes“) in den Ringsystemen des Saturn auf der Grundlage von Vielteilchen-Simulationen mit Eigengravitation und dissipativen Kollisionen. Für die Stoßzahl als Funktion der Geschwindigkeit verwendete Salo den in Gl. (8.11) gegebenen Zusammenhang von Bridges et al. und stellte fest, dass die genannten Wirbel - die schief zur orbitalen Bahn liegen und aus dem Zusammenspiel der Akkretion durch die Eigengravitation und der viskosen Scherung entstehen - im A- und B-Ring anzutreffen sein sollten. Diese Vorhersage bestätigten später Aufnahmen der Raumsonde „Cassini“ [79].

Ohtsuki und Emori [80] fanden heraus, dass eine hohe optische Dichte zur Bildung von gravitativen Instabilitäten und wakes führt. Daisaka et al. [81] stellten außerdem fest, dass die makroskopische Viskosität des Mediums durch diese Instabilitäten stark erhöht wird. Allerdings verwendeten beide Arbeiten in den numerischen Simulationen für alle Kollisionen eine konstante Stoßzahl. 
Auf der Grundlage des Kollisionsmodells aus Gl. (8.11) untersuchten Salo et al. [82] numerisch die Bildung viskoser Instabilitäten, die ebenfalls durch hohe optische Dichten erzeugt werden. Ballouz et al. [83] studierten den Zusammenhang zwischen wakes und viskosen Instabilitäten. Sie stellten fest, dass Systeme aus Teilchen mit ,glatten“ Oberflächen (d.h. wenig Reibung) zur Bildung von wakes tendieren, während die viskose Instabilität durch hohe Reibung zwischen den Partikeln begünstigt wird. Zur Modellierung der einzelnen Kontakte verwendeten die Autor*innen ein einfaches Feder-Dämpfer-Modell mit Reibung.

\subsection{Anwendungen im Sportbereich}

\subsubsection{Ballsportarten}

Ballsportarten ${ }^{17}$ sind ebenso vielfältig wie weit verbreitet. Die wissenschaftliche Untersuchung verschiedener Stoßprobleme im Bereich des Sports geschieht meistens vor dem Hintergrund, dass, gerade in Wettbewerbssportarten, die technischen Voraussetzungen (wie beispielsweise Bälle oder Schläger) für alle Spieler*innen durch Regeln standardisiert und vereinheitlicht werden sollen, damit nur die ,reine“ sportliche Leistung über Sieg und Niederlage entscheidet. Von der Seite der Wettkämpfer*innen besteht dagegen das Interesse, sich trotzdem im Rahmen des Reglements einen möglichst großen technologischen Vorteil zu verschaffen.

Zahlreiche Arbeiten existieren zur Physik der Kollisionen beim Tennis [84], Baseball [85], Golf [86], Cricket [87] und Hurling [88]. Dies betrifft sowohl den Kontakt zwischen Ball und Boden als auch den zwischen Ball und Schläger. Beides sind im Allgemeinen ebene Stöße mit Reibung einer Kugel auf eine gerade oder leicht gekrümmte Unterlage.

Die meisten wissenschaftlichen Untersuchungen der Stoßdynamik im Sport sind dabei experimenteller Natur. Bei der theoretischen Analyse gelangen bisher Starrkörper-Modelle [89] und FEM-basierte Modelle [90] zur Anwendung ${ }^{18}$. An dieser Stelle bieten kontaktmechanische Modelle eine einfache, aber robuste ,Zwischenlösung“, da Starrkörper-Modelle das dynamische Verhalten oft nur unzureichend erfassen ${ }^{19}$ und FEM-basierte Untersuchungen numerisch vergleichsweise aufwendig sind.

In den früheren Kapiteln stand die Kontaktmechanik massiver Körper im Mittelpunkt, die die Annahmen der Halbraumhypothese in ausreichender Näherung erfüllen. Aber inwieweit trifft das auf Sportbälle zu? Der Tennisball oder der Spielball beim Baseball deformieren sich während der Kollisionen sehr stark [93]; die meisten Sportbälle stellen außerdem anstatt

\footnotetext{
${ }^{17}$ Dies bezeichnet Sportarten, in denen Bälle eine wesentliche Rolle spielen; insofern fallen darunterentgegen der umgangssprachlichen Verwendung des Begriffs, die in der Regel auf Torspiele fokussiert ist - beispielsweise auch Golf oder Tennis.

${ }^{18}$ Eine gute Übersicht über analytische und FEM-basierte Modelle im Bereich des Baseball bietet die Arbeit von Cross [91].

${ }^{19}$ Für die Interaktion zwischen Schläger und Ball beim Cricket demonstrierten dies Allen et al. [92].
} 
Abb. 8.6 Rheologische Modelle für den Kontakt eines viskoelastischen Balls mit einer starren (a) oder nachgiebigen (b) Wand
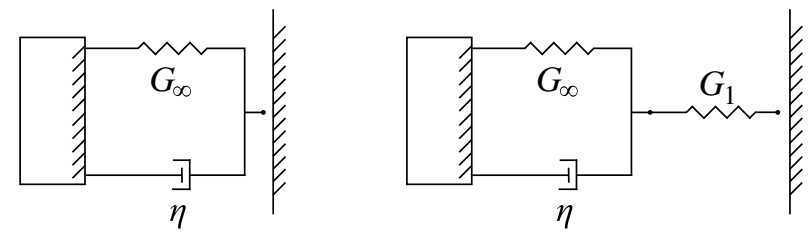

eines massiven Halbraums eigentlich eine mit Luft gefüllte Membran dar, deren Indentierung durch einen festen Körper eine grundsätzlich andere kontaktmechanische Aufgabe bildet. Allerdings greift man zur Modellierung des Balls häufig trotzdem auf einfache lineare viskoelastische oder viskoplastische Elemente zurück [94]; das Verhalten viskoelastischer rheologischer Elemente (wie des Kelvin-Voigt-Modells) in Stößen ist dabei im fünften und sechsten Kapitel dieses Buches ausführlich dargestellt.

Viele Effekte in den Kollisionen zwischen Sportbällen und festen Oberflächen sind durch solche rheologische Modelle einfach erklärbar. So merkte Cross [95] beispielsweise an, dass die Stoßzahl für die Kollision eines viskoelastischen Balls auf eine starre Wand kleiner ist als in dem Fall, dass die Wand eine elastische Nachgiebigkeit besitzt. Dies kann man sehr leicht mit den beiden in Abb. 8.6 dargestellten rheologischen Modellen verstehen. Der Ball werde durch ein Kelvin-Voigt-Element mit der Steifigkeit $G_{\infty}$ und der Viskosität $\eta$ dargestellt, die Wand sei starr oder elastisch mit dem Modul $G_{1}$.

Für den Fall einer starren Wand ist das in Gl.(5.57) eingeführte Dämpfungsmaß durch

$$
2 D=\frac{\operatorname{Im}\left[\hat{G}\left(\omega_{0}\right)\right]}{\operatorname{Re}\left[\hat{G}\left(\omega_{0}\right)\right]}=\frac{\eta \omega_{0}}{G_{\infty}},
$$

gegeben. Ist die Wand elastisch ${ }^{20}$, ergibt sich dagegen

$$
2 D=G_{1} \frac{\eta \omega_{0}}{G_{\infty}\left(G_{\infty}+G_{1}\right)+\left(\eta \omega_{0}\right)^{2}} .
$$

Man erkennt, dass das Dämpfungsmaß bei kleinen Frequenzen $\omega_{0}$ im Fall der elastischen Wand kleiner ist.

Ebenfalls von Cross [96] stammt der Vergleich des ebenen Stoßes unterschiedlicher Sportbälle auf eine starre Oberfläche mit den im sechsten Kapitel dargestellten theoretischen Vorhersagen von Maw et al. [97] für die Kollision mit einer elastischen Kugel. Die kontaktmechanische Theorie liefert dabei bessere Ergebnisse als entsprechende StarrkörperModelle, besonders gut ist die Übereinstimmung im Fall von Golfbällen (die den Annahmen der Halbraumhypothese auch vermutlich am nächsten kommen).

\footnotetext{
${ }^{20}$ Das entsprechende rheologische Element des Gesamtsystems aus Ball und Wand ist in diesem Fall eine - von der in Abb. 3.10 gezeigten Variante zwar abweichende aber äquivalente - Darstellung des viskoelastischen Standardkörpers.
} 
Bei der Interaktion zwischen Ball und Schläger kommen als die Analyse erschwerende Aspekte die Dynamik des Schlägers (einschließlich der Aktion der führenden Hand [98]) und beim Tennis die Kontakteigenschaften der Saiten-Bespannung hinzu.

\subsubsection{Schutzhelme}

Ein weiterer Forschungsbereich innerhalb des Sports, bei dem Kollisionen eine wesentliche Rolle spielen, ist die Auslegung von Schutzhelmen in Sportarten wie Football oder Eishockey. Diese Helme sind ebenfalls strikt reglementiert, um die Gesundheit der Spieler*innen bestmöglich zu schützen.

Die Sicherheitsstandards konzentrieren sich dabei vorrangig auf die Abfederung von translatorischen Beschleunigungen [99]; Schädelbrüche und ähnliche Kopfverletzungen sind dadurch in den fraglichen Sportarten selten. Dafür kommt es vergleichsweise oft zu leichten Schädel-Hirn-Traumata, wie beispielsweise Gehirnerschütterungen. Vor dem Hintergrund der im letzten Jahrzehnt verstärkten Diskussion möglicher neurologischer Spätfolgen von gehäuft auftretenden Gehirnerschütterungen ist daher die Frage, wie Schutzhelme ausgelegt sein müssen, um diese leichten Traumata zu vermeiden, in den wissenschaftlichen Fokus gerückt.

Eine kontaktmechanische Fragestellung ist dabei die Untersuchung der Wechselwirkung zwischen Stoßkörper und Helm sowie zwischen Helm und Kopf. Bei der theoretischen Analyse werden dabei in der Regel FEM-basierte Modelle verwendet [100].

Mehrere Studien kommen zu dem Ergebnis, dass laterale Stöße leichter zu Gehirnerschütterungen führen als frontale. Dies liegt einerseits an den auftretenden größeren Winkelbeschleunigungen um die Körperachse [101, 102]; andererseits führen laterale Kollisionen zu einer stärker lokalisierten Schädeldeformation und zu größeren Schubspannungen im Inneren des Gehirns [100].

\subsection{Anwendungen in der Medizin}

Nicht nur für Organe wie das Gehirn, sondern auch für Strukturen wie Knochen oder Gelenke stellen stoßartige Belastungen eine häufige und ernsthafte Schadensquelle dar. Als ,,stoßartig“ ist eine Last in diesem Zusammenhang nicht im Sinne der Kollision oder Einwirkung fester Teilchen zu verstehen, sondern als eine kurze Belastung mit sehr hohen Lastraten. So beträgt die Kraftänderung in Hüft- oder Kniegelenken bei einfachem Gehen bis zu $20 \mathrm{kN} / \mathrm{s}$ [103]. Beim Rennen steigert sich dieser Maximalwert noch einmal um den Faktor 10 [104].

Traumatische Belastungen von Gelenken führen dabei zu einem signifikant erhöhten Risiko, später an Arthrose des jeweiligen Gelenks zu erkranken [105]. Diese posttraumatische Arthrose betrifft häufig auch junge Erwachsene, für die ein künstliches Gelenk wegen der begrenzten Lebensdauer der Implantate - und der damit einhergehenden Notwendigkeit 
des erneuten Austauschs nach, in der Regel, etwa 15 bis 20 Jahren - keine wünschenswerte Therapieform darstellt. Was in diesem Zusammenhang als ,traumatische Belastung“ aufzufassen ist, ist eine in den letzten 10 Jahren sehr aktiv untersuchte Forschungsfrage.

$\mathrm{Zu}$ deren systematischer Beantwortung belasten Forscher*innen Knorpelgewebe, in vivo oder in vitro, durch einen einzelnen tatsächlichen Stoß mit einem parabolischen oder zylindrischen Gegenkörper von gegebener kinetischer Energie, die durch eine gespannte Feder [106] oder eine Fallgewichts-Apparatur [23] aufgebracht wird. Mit der Ermittlung der mechanischen Eigenschaften von Gelenkknorpel durch stoßbasierte Testverfahren hat sich dabei bereits der Abschn. 8.2.1 dieses Buches auseinandergesetzt. An dieser Stelle soll daher nur kurz zusammengefasst werden, welche medizinischen Erkenntnisse sich aus diesen Experimenten ergeben haben und welche Stellung die entsprechende Biokontaktmechanik in diesem Zusammenhang einnimmt.

Die Reaktion des Gewebes auf die mechanische Belastung gliedert sich in eine mechanische und eine anschließende biochemische Antwort des Systems (siehe die grobe schematische Darstellung in Abb. 8.7). Eine gute Übersicht über den Zusammenhang zwischen diesen beiden Stadien bietet die Arbeit von Natoli und Athanasiou [29]. Die mechanische Antwort besteht aus dem sich ergebenden Spannungs- und Verzerrungszustand im Inneren des Gewebes und eventuell bereits daraus resultierenden mechanischen Schädigungen, beispielsweise Rissen. Das angemessene Verständnis dieses Stadiums ist letztlich eine kontaktmechanische Aufgabe. Die Spannungen und Dehnungen setzen dann verschiedene, positive oder negative, zelluläre Prozesse (z. B. das Sterben von Chondrozyten [107]) in Gang, durch die das geschädigte Gewebe regeneriert oder degradiert.

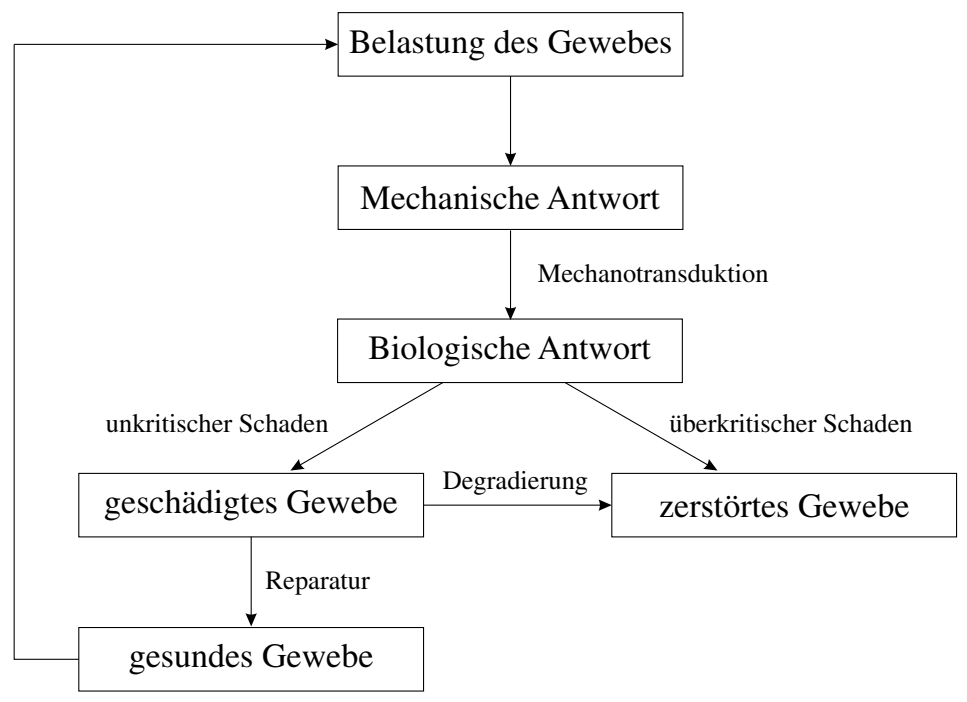

Abb. 8.7 Schematischer Ablauf der Reaktion von Knorpelgewebe auf eine mechanische Belastung (nach [29]) 
Es stellt sich die Frage, welche (einfachen) mechanischen Größen zur Charakterisierung der aus der stoßartigen Belastung folgenden Gewebeschädigung notwendig oder hinreichend sind. Eine schon längere Tradition hat dabei die Hypothese, dass die (vor dem Stoß vorhandene oder in der Kollision dissipierte) Energie am höchsten mit der Schädigung korreliert $^{21}$ [104, 105]. So genügt ein einzelner Stoß mit einer Energie von 0,28 J, um lokal Arthrose-typische Gewebe-Degradierung zu initiieren [106]. Eine Energie von 1,5 J führt bereits zur Bildung von Rissen ${ }^{22}$ [108]. Traumatische Belastungen mit sehr hohen Energien können sogar tief im Inneren des Gewebes zur Rissbildung führen [105]. Außerdem wurde berichtet, dass die Lebensfähigkeit der Chondrozyten linear mit der Stoßenergie abnimmt [110]. Als kritische Schwelle für den maximalen Kontaktdruck während der Belastung publizierten verschiedene Autor*innen Werte zwischen $13 \mathrm{MPa}$ [111] und 25 MPa [108]. Heiner et al. [112] untersuchten den Zusammenhang zwischen dem Frequenzspektrum der Kontaktkraft und akuten Verletzungen des Gewebes in Fallgewichtsversuchen und stellten fest, dass insbesondere die hohen Frequenzanteile mit der Gewebeschädigung korrelieren.

Bei großen Anregungsfrequenzen, beispielsweise durch Stöße, sind die zeitabhängigen Moduln von Gelenkknorpel nicht mehr deutlich kleiner als die des darunter liegenden Knochens [103]. Schwere stoßartige Belastungen führen deswegen eher zu einer Schädigung des Knochens als des Knorpels [103]. Ein geschädigter subchondraler Knochen stört in der Folge allerdings auch den Stoffwechsel des Knorpelgewebes [113].

Die bisher einzige Arbeit, in der die mechanische und biochemische Reaktion des Gewebes auf die stoßartige Belastung in einem gemeinsamen, gekoppelten mathematischen Modell untersucht wurden, ist die Publikation von Kapitanov et al. [114]. Die Autor*innen verwendeten zur Behandlung des Kontaktproblems ein FEM-basiertes Modell; das Knorpelgewebe modellierten sie dabei allerdings als linear-elastisches Medium. Dies ist eine sehr grobe Vereinfachung des tatsächlichen Materialverhaltens. Hier besteht also noch sehr viel Spielraum für kontaktmechanisch rigorosere Modelle; in diesem Zusammenhang sei abschließend auf die hervorragende Monografie von Argatov und Mishuris [115] zur Kontaktmechanik von Knorpelgewebe hingewiesen.

\subsection{Zusammenfassung}

Stöße treten häufiger in technischen, biologischen oder biotechnologischen Systemen auf, als man denkt. Da jede kurze Belastung mit sehr großen Lastraten „stoßartig“ ist, und es mithin keine Rolle spielt, ob sie zwischen unverbundenen Teilen eines Systems (wie bei klassischen Kollisionen) oder zwischen verbundenen Komponenten (wie bei Gelenken) auftritt, sind Stöße sehr allgemeine Prozesse, die in einer Vielzahl physikalischer, technischer oder medizinischer Anwendungen von Bedeutung sind.

\footnotetext{
${ }^{21}$ Das passt auch zu energiebasierten Verschleißgesetzen für technische Systeme, siehe Abschn. 8.1.

${ }^{22}$ Diese Risse sind in einem Winkel von $45^{\circ}$ orientiert [108]. Das spricht dafür, dass die maximale Scherspannung für ihre Bildung und Ausbreitung bestimmend ist [109].
} 
Im vergangenen Kapitel wurde eine Auswahl dieser Anwendungen kurz diskutiert. Da jedes behandelte Themengebiet einen eigenen Forschungszweig darstellt, kann die Darstellung selbstverständlich keinen Anspruch auf Vollständigkeit erheben, auch nicht als Übersicht. Stattdessen war das Ziel dieses Kapitels, zu skizzieren, an welchen Stellen die in den früheren Teilen dieses Buches entwickelten Methoden und erhaltenen Ergebnisse für bestimmte Anwendungsgebiete relevant sein können.

Zunächst ist die einmalige oder wiederholte stoßartige Einwirkung auf die Oberfläche eines - organischen oder anorganischen - Materials eine Quelle von Schädigungen durch Verschleiß und Rissbildung. Da dabei häufig abrasive Mechanismen dominieren, kann man oft, sowohl für metallische Oberflächen als auch für Knorpelgewebe, einfache energiebasierte Verschleißgesetze formulieren. Die Energiedissipation bei der Belastung ergibt sich dabei direkt aus der kontaktmechanischen Stoßtheorie.

Andererseits sind stoßbasierte Tests einfache schnelle Verfahren zur Bestimmung von mechanischen Materialeigenschaften unter dynamischer Belastung. Sowohl für viskoelastische als auch für elasto-plastische Medien lässt sich dabei die in dem Versuch gemessene Stoßzahl, zumindest im quasistatischen Fall, mit einer bestimmten Materialkenngröße in Verbindung bringen.

Theorie und Anwendungen der Mechanik granularer Medien sind ein riesiges Forschungsgebiet, das wesentlich auf der korrekten Beschreibung der Wechselwirkung zwischen zwei Teilchen des granularen Materials beruht. Dabei spielt besonders die Geschwindigkeitsabhängigkeit der Stoßzahlen eine große Rolle, sowohl für die Komplexität der theoretischen Beschreibung als auch für die langfristige Dynamik des granularen Mediums.

Zur numerischen Simulation granularer Materialien bedient man sich oft der DiskreteElemente-Methode (DEM). In der zeitgesteuerten DEM muss man explizite Kraftgesetze für die Wechselwirkungen zwischen den einzelnen Elementen formulieren. Da mechanische Kontakte, z. B. durch Reibung oder inelastische Deformationen, allerdings von der Belastungsgeschichte abhängen, ist dies nur schwierig auf rigorose Art möglich. Dieses Problem kann für ausreichend dünne ${ }^{23}$ Systeme durch die Simulation entlang diskreter Ereignisse (Stöße) umgangen werden, die durch die Angabe der Stoßzahlen vollständig beschreibbar sind. Die korrekte Geschwindigkeitsabhängigkeit der Stoßzahlen ergibt sich wiederum aus der kontaktmechanischen Stoßtheorie.

Planetare Ringsysteme sind ein populäres Beispiel granularer Medien. Wie für alle granularen Systeme hängt die Dynamik und insbesondere die Stabilität der Ringe maßgeblich von der einzelnen Interaktion zwischen zwei Teilchen des Ringsystems ab. Zur Beschreibung dieser Interaktionen sind viskoelastische Ansätze und Fragmentierungsmodelle gut geeignet.

Im Sport treten relevante Kollisionen offensichtlich bei Ballsportarten auf. Sportbälle sind häufig gefüllte Membranen, die eine etwas andere Kontaktmechanik aufweisen als

\footnotetext{
${ }^{23}$ im Sinne der Konzentration
} 
massive Körper. Allerdings verwendet man zur Charakterisierung ihrer Kontakteigenschaften oft rheologische Modelle, deren Kontaktmechanik in dem vorliegenden Buch ausführlich diskutiert wurde.

Aber auch in anderen Sportarten, wenn die Gesundheit der Spieler*innen durch stoßartige Einwirkungen, beispielsweise im Kopfbereich, gefährdet sein kann, ist das korrekte Verständnis des Stoßvorgangs eine wichtige Grundlage, unter anderem zur Auslegung von Schutzhelmen.

In Gelenken steigt durch traumatische Belastungen und damit einhergehende Schädigungen des Knorpelgewebes das Arthrose-Risiko. Die biomechanische Reaktion des Gewebes auf die stoßartige Belastung besteht aus einer mechanischen und einer daraus resultierenden biochemischen Antwort. Zur Beschreibung des mechanischen Aspekts ist eine saubere Beschreibung der Kontakt-Wechselwirkung eine unabdingbare Voraussetzung.

\section{Literatur}

1. Tarbe, R., \& Kulu, P. (2008) Impact wear tester for the study of abrasive erosion and milling processes. In 6th International DAAAM Baltic Conference Industrial Engineering, Tallin.

2. Ellermaa, R. R. R. (1993). Erosion prediction of puremetals and carbon steels. Wear, 162164(B), 1114-1122.

3. Molinari, J. F., \& Ortiz, M. (2002). A study of solid-particle erosion of metallic targets. International Journal of Impact Engineering, 27(4), 347-358.

4. Walley, S. M., Field, J. E., \& Yennadhiou, P. (1984). Single solid particle impact erosion damage on polypropylene. Wear, 100(1-3), 263-280.

5. Arnold, J. C., \& Hutchings, I. M. (1989). Flux rate effects in the erosive wear of elastomers. Journal of Materials Science, 24(3), 833-839.

6. Tewari, U. S., Harsha, A. P., Häger, A. M., \& Friedrich, K. (2003). Solid particle erosion of carbon fibre- and glass fibre-epoxy composites. Composites Science and Technology, 63(3-4), 549-557.

7. Arjula, S., Harsha, A. P., \& Ghosh, M. K. (2008). Solid-particle erosion behavior of highperformance thermoplastic polymers. Journal of Materials Science, 43(6), 1757-1768.

8. Kleis, I., \& Kulu, P. (2008). Solid particle erosion. London: Springer.

9. Finnie, I. (1960). Erosion of surfaces by solid particles. Wear, 3(2), 87-103.

10. Beckmann, G., \& Gotzmann, J. (1981). Analytical model of the blast wear intensity of metals based on a general arrangement for abrasive wear. Wear, 73(2), 325-353.

11. Hutchings, I. M., Winter, R. E., \& Field, J. E. (1976). Solid particle erosion of metals: The removal of surface material by spherical projectiles. Proceedings of the Royal Society of London, Series A, 348, 379-392.

12. Evans, A. G., Gulden, M. E., \& Rosenblatt, M. (1978). Impact damage in brittle materials in the elasticplastic response regime. Proceedings of the Royal Society of London, Series A, 361, 343-365.

13. Khrushchov, M. M., \& Babichev, M. A. (1960). Investigation of wear of metals. Moskau: Russian Academy of Sciences.

14. Honda, K., \& Yamada, K. (1925). Some experiments on the abrasion of metals. Journal of the Institute of Metals, 33(1), 49-68.

15. Brach, R. M. (1988). Impact dynamicswith applications to solid particle erosion. International Journal of Impact Engineering, 7(1), 37-53. 
16. Souilliart, T., Rigaud, E., Le Bot, A., \& Phalippou, C. (2017). Energy-based wear law for oblique impacts in dry environment. Tribology International, 105, 241-249.

17. Field, J. E., Walley, S. M., Proud, W. G., Goldrein, H. T., \& Siviour, C. R. (2004). Review of experimental techniques for high rate deformation and shock studies. International Journal of Impact Engineering, 30(7), 725-775.

18. Bassi, A. C. (1978). Dynamic modulus of rubber by impact and rebound measurements. Polymer Engineering and Science, 18(10), 750-754.

19. Taylor, G. I. (1948). The use of flat-ended projectiles for determining dynamic yield stress I. Theoretical considerations. Proceedings of the Royal Society of London, Series A, 194, 289-299.

20. Jones, S. E., Drinkard, J. A., Rule, W. K., \& Wilson, L. L. (1998). An elementary theory for the Taylor impact test. International Journal of Impact Engineering, 21(1-2), 1-13.

21. Lu, G., Wang, B., \& Zhang, T. (2001). Taylor impact test for ductile porous materials - Part 1: Theory. International Journal of Impact Engineering, 25(10), 981-991.

22. Sarva, S., Mulliken, A. D., \& Boyce, M. C. (2007). Mechanics of Taylor impact testing of polycarbonate. International Journal of Solids and Structures, 44(7-8), 2381-2400.

23. Burgin, L. V., \& Aspden, R. M. (2007). A drop tower for controlled impact testing of biological tissues. Medical Engineering \& Physics, 29(4), 525-530.

24. Kang, W., Chen, Y. C., Bagchi, A., \& O'Shaughnessy, T. J. (2017). Characterization and detection of acceleration-induced cavitation in soft materials using a drop-tower-based integrated system. Review of Scientific Instruments, 88(12), 125113. https://doi.org/10.1063/1.5000512.

25. Ruta, P., \& Szydło, A. (2005). Drop-weight test based identification of elastic half-space model parameters. Journal of Sound and Vibration, 282(1-2), 411-427.

26. Popov, V. L., Heß, M., \& Willert, E. (2018). Handbuch der Kontaktmechanik. Exakte Lösungen axialsymmetrischer Kontaktprobleme. Berlin: Springer Vieweg.

27. Burgin, L. V., Edelsten, L., \& Aspden, R. M. (2014). The mechanical and material properties of elderly human articular cartilage subject to impact and slow loading. Medical Engineering \& Physics, 36(2), 226-232.

28. Pearson, B., \& Espino, D. M. (2013). Effect of hydration on the frequency-dependent viscoelastic properties of articular cartilage. Proceedings of the Institution of Mechanical Engineers Part H: Journal of Engineering in Medicine, 227(11), 1246-1252.

29. Natoli, R. M., \& Athanasiou, K. A. (2009). Traumatic loading of articular cartilage: Mechanical and biological responses and post-injury treatment. Biorheology, 46(6), 451-485.

30. Selyutina, N. S., Argatov, I. I., \& Mishuris, G. S. (2015). On application of Fung's quasilinear viscoelastic model to modeling of impact experiments for articular cartilage. Mechanics Research Communications, 67, 24-30.

31. Springhetti, R., \& Selyutina, N. S. (2018). Viscoelastic modeling of articular cartilage under impact loading. Meccanica, 53(3), 519-530.

32. Pierce, D. M., Unterberger, M. J., Trobin, W., Ricken, T., \& Holzapfel, G. A. (2016). Amicrostructurally based continuum model of cartilage viscoelasticity and permeability incorporating measured statistical fiber orientations. Biomechanics and Modeling in Mechanobiology, 15(1), 229-244.

33. Nega, A., Nikraz, H., \& Al-Qadi, I. L. (2016). Dynamic analysis of fallingweight deflectometer. Journal of Traffic and Transportation Engineering, 3(5), 427-437.

34. Ruta, P., Krawczyk, B., \& Szydło, A. (2015). Identification of pavement elastic moduli by means of impact test. Engineering Structures, 100, 201-211.

35. Aiello, M., Galvanetto, U., \& Iannucci, L. (2007). Numerical simulations of motorcycle helmet impact tests. International Journal of Crashworthiness, 12(1), 1-7.

36. Mills, N. J., Wilkes, S., Derler, S., \& Flisch, A. (2009). FEA of oblique impact tests on a motorcycle helmet. International Journal of Impact Engineering, 36(7), 913-925. 
37. Jaeger, H. M., Nagel, S. R., \& Behringer, R. P. (1996). Granular solids, liquids, and gases. Reviews of Modern Physics, 68(4), 1259-1273.

38. Andreotti, B., Forterre, Y., \& Pouliquen, O. (2013). Granular media: Between fluid and solid. Cambridge: Cambridge University Press.

39. Lun, C. K. K., \& Savage, S. B. (1987). A simple kinetic theory for granular flow of rough, inelastic, spherical particles. Journal of Applied Mechanics, 54(1), 47-53.

40. Campbell, C. S. (1990). Rapid granular flows. Annular Review of Fluid Mechanics, 22(1), 57-92.

41. van Noije, T. P. C., \& Ernst, M. H. (1998). Velocity distributions in homogeneous granular fluids: The free and the heated case. Granular Matter, 1(2), 57-64.

42. Bar-Lev, O. (2005). Kinetic and hydrodynamic theory of granular gases. dissertation, Tel Aviv University.

43. Walton, O. R., \& Braun, R. L. (1986). Viscosity, granular-temperature, and stress calculations for shearing assemblies of inelastic, frictional disks. Journal of Rheology, 30(5), 949-980.

44. Lun, C. K. K., \& Savage, S. B. (1986). The effects of an impact velocity dependent coefficient of restitution on stresses developed by sheared granular materials. Acta Mechanica, 63(1-4), $15-44$.

45. Brilliantov, N. V., \& Pöschel, T. (2000). Velocity distribution in granular gases of viscoelastic particles. Physical Review E, 61(5B), 5573-5587.

46. Schwager, T., \& Pöschel, T. (1998). Coefficient of normal restitution of viscous particles and cooling rate of granular gases. Physical Review E, 57(1), 650-654.

47. Dubey, A. K., Brodova, A., Puri, S., \& Brilliantov, N. V. (2013). Velocity distribution function and effective restitution coefficient for a granular gas of viscoelastic particles. Physical Review E, 87(6), 062202. https://doi.org/10.1103/PhysRevE.87.062202.

48. Pöschel, T., Brilliantov, N. V., \& Schwager, T. (2002). Violation of molecular chaos in dissipative gases. International Journal of Modern Physics C, 13(9), 1263-1272.

49. Pöschel, T., \& Schwager, T. (2005). Computational granular dynamics: Models and algorithms. Berlin: Springer.

50. Zohdi, T. I. (2007). An introduction to modeling and simulation of particulate flows. Philadelphia: SIAM Society for Industrial und Applied Mathematics.

51. Kačianauskas, R., Kruggel-Emden, H., Markauskas, D., \& Zdancevičius, E. (2015). Critical assessment of visco-elastic damping models used in DEM simulations. Procedia Engineering, 102, 1415-1425.

52. Lubachevsky, B. D. (1991). How to simulate billiards and similar systems. Journal of Computational Physics, 94(2), 255-283.

53. Goldhirsch, I. I. (2001). Granular gases: Probing the boundaries of hydrodynamics. In T. von Pöschel \& S. Luding (Hrsg.), Granular gases (S. 79-99). Berlin: Springer.

54. Haff, P. K. (1983). Grain flow as a fluid-mechanical phenomenon. Journal of Fluid Mechanics, 134, 401-430.

55. Goldhirsch, I. I., \& Zanetti, G. (1993). Clustering instability in dissipative gases. Physical Review Letters, 70(11), 1619-1622.

56. Falcon, E., Fauve, S., \& Laroche, C. (2001). Experimental study of a granular gas fluidized by vibrations. In T. von Pöschel \& S. Luding (Hrsg.), Granular gases (S. 244-253). Berlin: Springer.

57. Schanz, T. (Hrsg.). (2007). Theoretical and numerical unsaturated soil mechanics. Berlin: Springer.

58. Savage, S. B. (1998). Modeling and granular material boundary value problems. In H. J. von Herrman, J. P. Hovi, \& S. Luding (Hrsg.), Physics of dry granular media (S. 25-95). Dordrecht: Kluwer. 
59. Savage, S. B. (1997). Problems in the statics and dynamics of granular materials. In R. P. von Behringer \& J. T. Jenkins (Hrsg.), Proceedings of the third International Conference on Powders \& Grains, Durham, North Carolina, 18-23 May 1997. Rotterdam: A.A. Balkema Publishers.

60. Nesterenko, V. F. (1983). Propagation of nonlinear compression pulses in granular media. Journal of Applied Mechanics and Technical Physics, 24(5), 733-743.

61. Job, S., Melo, F., Sokolow, A., \& Sen, S. (2007). Solitarywave trains in granular chains: Experiments, theory and simulations. Granular Matter, 10(1), 13-20.

62. Sen, S., Hong, J., Bang, J., Avalos, E., \& Doney, R. (2008). Solitary waves in the granular chain. Physics Reports, 462(2), 21-66.

63. Job, S., Melo, F., Sokolow, A., \& Sen, S. (2005). How hertzian solitary waves interact with boundaries in a 1D granular medium. Physical Review Letters, 94(17), 178002. https://doi.org/ 10.1103/PhysRevLett.94.178002.

64. Daraio, C., Nesterenko, V. F., Herbold, E. B., \& Jin, S. (2006). Tunability of solitary wave properties in onedimensional strongly nonlinear phononic crystals. Physical Review E, 73, 026610. https://doi.org/10.1103/PhysRevE.73.026610.

65. On, T., LaVigne, P. A., \& Lambros, J. (2014). Development of plastic nonlinear waves in one-dimensional ductile granular chains under impact loading. Mechanics of Materials, 68, 29-37.

66. Pöschel, T., \& Brilliantov, N. V. (2001). Chains of viscoelastic spheres. In T. von Pöschel \& S. Luding (Hrsg.), Granular gases (S. 203-212). Berlin: Springer.

67. Bridges, F. G., Hatzes, A., \& Lin, D. N. C. (1984). Structure, stability and evolution of Saturn's rings. Nature, 309, 333-335.

68. Hatzes, A. P., Bridges, F. G., \& Lin, D. N. C. (1988). Collisional properties of ice spheres at low impact velocities. Monthly Notices of the Royal Astronomical Society, 231(4), 1091-1115.

69. Hatzes, A. P., Bridges, F. G., Lin, D. N. C., \& Sachtjen, S. (1991). Coagulation of particles in Saturn's rings: Measurements of the cohesive force of water frost. Icarus, 89(1), 113-121.

70. Higa, M., Arakawa, M., \& Maeno, N. (1996). Measurements of restitution coefficients of ice at low temperatures. Planetary and Space Science, 44(9), 917-925.

71. Gorkavy, N. N., \& Fridmann, A. M. (1990). The physics of planetary rings. Soviet Physics Uspekhi, 33(2), 95-133.

72. Spahn, F., Hertzsch, J. M., \& Brilliantov, N. V. (1995). The role of particle collisions for the dynamics in planetary rings. Chaos, Solitons \& Fractals, 5(10), 1945-1964.

73. Brilliantov, N. V., Spahn, F., Hertzsch, J. M., \& Pöschel, T. (1996). Model for collisions in granular gases. Physical Review E, 53(5), 5382-5392.

74. Lynden-Bell, D., \& Pringle, J. E. (1974). The evolution of viscous discs and the origin of the nebular variables. Monthly Notices of the Royal Astronomical Society, 168(3), 603-637.

75. Goldreich, P., \& Tremaine, S. (1978). The velocity dispersion in Saturn's rings. Icarus, 34(2), 227-239.

76. Borderies, N., Goldreich, P., \& Tremaine, S. (1985). A granular flow model for dense planetary rings. Icarus, 63(3), 406-420.

77. Lin, D. N. C., \& Bodenheimer, P. (1981). On the stability of saturn's rings. The Astrophysical Journal, 248(1), L83-L86.

78. Salo, H. (1992). Gravitational wakes in saturn's rings. Nature, 359, 619-621.

79. Salo, H., Karjalainen, R., \& French, R. G. (2004). Photometric modeling of saturn's rings. II. Azimuthal asymmetry in reflected and transmitted light. Icarus, 170(1), 70-90.

80. Ohtsuki, K., \& Emori, H. (2000). Local N-Body simulations for the distribution and evolution of particle velocities in planetary rings. The Astronomical Journal, 119(1), 403-416.

81. Daisaka, H., Tanaka, H., \& Ida, S. (2001). Viscosity in a dense planetary ring with selfgravitating particles. Icarus, 154(2), 296-312. 
82. Salo, H., Schmidt, J., \& Spahn, F. (2001). Viscous overstability in saturn's B ring: I. Direct simulations and measurement of transport coefficients. Icarus, 153(2), 295-315.

83. Ballouz, R. L., Richardson, D. C., \& Morishima, R. (2017). Numerical simulations of saturn's B ring: Granular friction as a mediator between self-gravity wakes and viscous overstability. The Astronomical Journal, 153(4), 146. https://doi.org/10.3847/1538-3881/aa60be.

84. Cross, R. (2003). Oblique impact of a tennis ball on the strings of a tennis racket. Sports Engineering, 6(4), 235-254.

85. Nathan, A. M. (2003). Characterizing the performance of baseball bats. American Journal of Physics, 71(2), 134-143.

86. Arakawa, K. (2017). An analytical model of dynamic sliding friction during impact. Scientific Reports, 7, 40102. https://doi.org/10.1038/srep40102.

87. James, D., Curtis, D., Allen, T., \& Rippin, T. (2012). The validity of a rigid body model of a cricket ball-bat impact. Procedia Engineering, 34, 682-687.

88. Collins, F., Brabazon, D., \& Moran, K. (2011). Viscoelastic impact characterisation of solid sports balls used in the Irish sport of hurling. Sports Engineering, 14(1), 15-25.

89. Ghaednia, H., Cermik, O., \& Marghitu, D. B. (2015). Experimental and theoretical study of the oblique impact of a tennis ball with a racket. Proceedings of the Institution of Mechanical Engineers, Part P: Journal of Sports Engineering and Technology, 229(3), 149-158.

90. Allen, T., Haake, S. J., \& Goodwill, S. R. (2010). Effect of friction on tennis ball impacts. Proceedings of the Institution of Mechanical Engineers, Part P: Journal of Sports Engineering and Technology, 224(3), 229-236.

91. Cross, R. (2014). Impact of sports balls with striking implements. Sports Engineering, 17(1), $3-22$.

92. Allen, T., Fauteux-Brault, O., James, D., \& Curtis, D. (2014). Finite elementmodel of a cricket ball impacting a bat. Procedia Engineering, 72, 521-526.

93. Cross, R. (1999). The bounce of a ball. American Journal of Physics, 67(3), 222-227.

94. Ismail, K. A., \& Stronge, W. J. (2012). Viscoplastic analysis for direct impact of sports balls. International Journal of Non-Linear Mechanics, 47(4), 16-21.

95. Cross, R. (2000). The coefficient of restitution for collisions of happy balls, unhappy balls, and tennis balls. American Journal of Physics, 68(11), 1025-1031.

96. Cross, R. (2002). Grip-slip behavior of a bouncing ball. American Journal of Physics, 70(11), 1093-1102.

97. Maw, N., Barber, J. R., \& Fawcett, J. N. (1976). The oblique impact of elastic spheres. Wear, 38(1), 101114.

98. Cross, R. (1999). Impact of a ball with a bat or racket. American Journal of Physics, 67(8), $692-702$.

99. Ouckama, R., \& Pearsall, D. J. (2012). Impact performance of ice hockey helmets: Head acceleration versus focal force dispersion. Proceedings of the Institution of Mechanical Engineers, Part P: Journal of Sports Engineering and Technology, 226(3-4), 185-192.

100. Zhang, L., Yang, K. H., \& King, A. I. (2001). Comparison of brain responses between frontal and lateral impacts by finite element modeling. Journal of Neurotrauma, 18(1), 21-30.

101. McIntosh, A. S., et al. (2014). The biomechanics of concussion in unhelmeted football players in Australia: A case-control study. BMJ Open, 4(5), e005078. https://doi.org/10.1136/bmjopen2014-005078.

102. Rowson, S., et al. (2012). Rotational head kinematics in football impacts: An injury risk function for concussion. Annals of Biomedical Engineering, 40(1), 1-13.

103. Burgin, L. V., \& Aspden, R. M. (2008). Impact testing to determine the mechanical properties of articular cartilage in isolation and on bone. Journal of Materials Science: Materials in Medicine, 19(2), 703-711. 
104. Kos, P., et al. (2011). Correlation of dynamic impact testing, histopathology and visual macroscopic assessment in human osteoarthritic cartilage. International Orthopaedics, 35(11), 17331739 .

105. Anderson, D. D., et al. (2011). Post-traumatic osteoarthritis: Improved understanding and opportunities for early intervention. Journal of Orthopaedic Research, 29(6), 802-809.

106. Alexander, P. G., et al. (2012). An in vivo lapine Model for impact-induced injury and osteoarthritic degeneration of articular cartilage. Cartilage, 3(4), 323-333.

107. Ding, L., et al. (2010). Mechanical impact induces cartilage degradation via mitogen activated protein kinases. Osteoarthritis Cartilage, 18(11), 1509-1517.

108. Verteramo, A., \& Seedhom, B. B. (2007). Effect of a single impact loading on the structure and mechanical properties of articular cartilage. Journal of Biomechanics, 40(16), 3580-3589.

109. Atkinson, T. S., Haut, R. C., \& Altiero, N. J. (1998). Impact-Induced fissuring of articular cartilage: An investigation of failure criteria. Journal of Biomechanical Engineering, 120(2), 181-187.

110. Jeffrey, J. E., Gregory, D. W., \& Aspden, R. M. (1995). Matrix damage and chondrocyte viability following a single impact load on articular cartilage. Archives of Biochemistry and Biophysics, 322(1), 87-96.

111. Bonnevie, E. D., et al. (2015). Characterization of tissue response to impact loads delivered using a hand-held instrument for studying articular cartilage injury. Cartilage, 6(4), 226-232.

112. Heiner, A. D., et al. (2012). Frequency content of cartilage impact force signal reflects acute histologic structural damage. Cartilage, 3(4), 314-322.

113. Lin, Y. Y., et al. (2009). The mandibular cartilage metabolism is altered by damaged subchondral bone from traumatic impact loading. Annals of Biomedical Engineering, 37(7), 1358-1367.

114. Kapitanov, G. I., Wang, X., Ayati, B. P., Brouillette, M. J., \& Martin, J. A. (2016). Linking cellular and mechanical processes in articular cartilage lesion formation: A mathematical model. Frontiers in Bioengineering and Biotechnology, 4, 80. https://doi.org/10.3389/fbioe. 2016.00080.

115. Argatov, I. I., \& Mishuris, G. S. (2015). Contact mechanics of articular cartilage layers: Asymptotic models (S. 164). Basel: Springer International Publishing.

Open Access Dieses Kapitel wird unter der Creative Commons Namensnennung 4.0 International Lizenz (http://creativecommons.org/licenses/by/4.0/deed.de) veröffentlicht, welche die Nutzung, Vervielfältigung, Bearbeitung, Verbreitung und Wiedergabe in jeglichem Medium und Format erlaubt, sofern Sie den/die ursprünglichen Autor(en) und die Quelle ordnungsgemäß nennen, einen Link zur Creative Commons Lizenz beifügen und angeben, ob Änderungen vorgenommen wurden.

Die in diesem Kapitel enthaltenen Bilder und sonstiges Drittmaterial unterliegen ebenfalls der genannten Creative Commons Lizenz, sofern sich aus der Abbildungslegende nichts anderes ergibt. Sofern das betreffende Material nicht unter der genannten Creative Commons Lizenz steht und die betreffende Handlung nicht nach gesetzlichen Vorschriften erlaubt ist, ist für die oben aufgeführten Weiterverwendungen des Materials die Einwilligung des jeweiligen Rechteinhabers einzuholen. 